\title{
Transposition
}

Musique et Sciences Sociales

\section{Après les attaques terroristes de l'État islamique à Paris. Enquête sur les rapports entre musique, propagande et violence armée}

After the Islamic State terrorist attacks in Paris. On music, propaganda and armed violence

\section{Luis Velasco-Pufleau}

\section{OpenEdition \\ Journals}

Édition électronique

URL : http://journals.openedition.org/transposition/1327

DOI : 10.4000/transposition. 1327

ISSN : 2110-6134

Éditeur

CRAL - Centre de recherche sur les arts et le langage

\section{Référence électronique}

Luis Velasco-Pufleau, «Après les attaques terroristes de l'État islamique à Paris. Enquête sur les

rapports entre musique, propagande et violence armée », Transposition [En ligne], 5 | 2015, mis en ligne le 15 décembre 2015, consulté le 10 décembre 2020. URL : http://journals.openedition.org/

transposition/1327 ; DOI : https://doi.org/10.4000/transposition.1327

Ce document a été généré automatiquement le 10 décembre 2020.

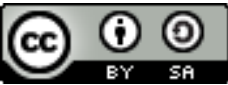

La revue Transposition est mise à disposition selon les termes de la Licence Creative Commons Attribution - Partage dans les Mêmes Conditions 4.0 International. 


\title{
Après les attaques terroristes de l'État islamique à Paris. Enquête sur les rapports entre musique, propagande et violence armée
}

\author{
After the Islamic State terrorist attacks in Paris. On music, propaganda and \\ armed violence
}

Luis Velasco-Pufleau

\section{Introduction : musique, propagande et violence}

1 Lors de la série d'attaques terroristes simultanées qui ont eu lieu à Paris le vendredi 13 novembre 2015, trois combattants de l'État islamique prennent d'assaut la salle de concert du Bataclan où se tenait un concert de rock ${ }^{1}$. L'attaque fait 90 victimes civiles non armées et de nombreux blessés. La plupart des combattants de l'État islamique qui ont pris part aux attaques sont des Européens francophones, qui sont nés et ont grandi en France ou en Belgique. La stupeur et le choc ont été immenses. Dans le communiqué audio de revendication des attentats ${ }^{2}$, le porte-parole de l'organisation terroriste explique, en français, les raisons de ces attaques :

Dans une attaque bénie dont Allah a facilité les causes, un groupe de croyants, des soldats du Califat - qu'Allah leur donne puissance et victoire - a pris pour cible la capitale des abominations et de la perversion, celle qui porte la bannière de la croix en Europe : Paris. Un groupe ayant divorcé la vie d'ici-bas s'est avancé vers leur ennemi, cherchant la mort dans le sentier d'Allah, secourant sa religion, son prophète et ses alliés, et voulant humilier ses ennemis. Ils ont été véridiques avec Allah, nous les considérons comme tels. Allah a conquis par leur main et a jeté la crainte dans le cœur des croisés dans leur propre terre.

Huit frères, portant des ceintures d'explosifs et des fusils d'assaut, ont pris pour cible des endroits choisis minutieusement à l'avance, au cœur de la capitale française: le Stade de France, lors du match de deux pays croisés, la France et 
l'Allemagne, auquel assistait l'imbécile de France : François Hollande ; le Bataclan, où étaient ressemblés des centaines d'idolâtres dans une fête de perversité ; ainsi que d'autres cibles dans le $10^{\mathrm{e}}$, le $11^{\mathrm{e}}$ et le $18^{\mathrm{e}}$ arrondissements. Et ce, simultanément. [...] La France et ceux qui suivent sa voie doivent savoir qu'ils restent les principales cibles de l'État Islamique. Qu'ils continueront à sentir l'odeur de la mort pour avoir pris la tête de la croisade, avoir osé insulter notre prophète, s'être vantés de combattre l'islam en France et frapper les musulmans en terre du Califat avec leurs avions, qui ne leur ont servi à rien dans les rues malodorantes de Paris. Cette attaque n'est que le début de la tempête et un avertissement pour ceux qui veulent méditer et tirer des leçons ${ }^{3}$.

2 Les raisons évoquées dans le communiqué pour justifier les attentats sont clairement politiques, présentant ceux-ci comme des représailles à des actions spécifiques engagées par la France. Cela fait partie de la stratégie de l'État islamique «d'humilier et d'épuiser » l'ennemi, de sa politique de «faire payer le prix » afin de "répandre le désespoir dans le cœur de l'ennemi $»^{4}$. Le président François Hollande qualifie aussitôt les attaques d'« acte de guerre qui a été commis par une armée terroriste, Daesh ${ }^{5}$, une armée djihadiste, contre la France, contre les valeurs que nous défendons partout dans le monde, contre ce que nous sommes: un pays libre qui parle à l'ensemble de la planète $»^{6}$. Dès le lendemain des attentats, les médias français proposent aussi des analyses et des interprétations extrêmement diverses. Sur les ondes d'une radio nationale (France Culture), une émission prend pour titre la question "Que nous veut Daesh ? $»^{7}$. De nombreuses tribunes sont publiées dans la presse. Certains avancent l'hypothèse que les terroristes s'en prennent surtout aux valeurs des démocraties libérales ou à la "liberté culturelle $»^{8}$. D'autres affirment qu'un "mode de vie a été assassiné : hédonisme, émotions partagées, mélange de culture et d'ivresse " ${ }^{9}$. Pour certains intellectuels, par exemple, les ressorts de cette " guerre » seraient avant tout économiques ou la réaction au déracinement social et à un défaut de reconnaissance ${ }^{10}$.

Néanmoins, très peu de réponses ont été avancées concernant le fait que la musique en général, et un concert de rock en particulier (qualifié de "fête de perversité »), ait été une des cibles choisies "minutieusement à l'avance " par l'État islamique ${ }^{11}$. Lors de l'attentat du Bataclan, les assaillants s'en sont pris en premier lieu aux personnes qui écoutaient la musique et non aux musiciens. Comme l'a constaté Julian Dorio, l'un des musiciens du groupe, dès le début de la fusillade les terroristes « tiraient sans relâche sur les spectateurs $»^{12}$. Bien que l'un des objectifs principaux des attentats simultanés était de faire le plus grand nombre de victimes possible - tout particulièrement l'attentat manqué contre le Stade de France -, lors de l'attaque du Bataclan c'est aussi l'écoute de la musique qui a été visée ${ }^{13}$. De ce fait, il est nécessaire d'interroger les liens entre cet acte hautement symbolique, le discours salafiste ${ }^{14}$ sur la musique (qui fait partie du corpus idéologique de l'État islamique) et l'utilisation que cette organisation djihadiste fait du chant dans sa propre propagande ${ }^{15}$. Car, comme le philosophe Philippe-Joseph Salazar l'affirme, nous sommes bien dans « une guerre asymétrique de propagande, sur tous les chefs, de tous les poids $»^{16}$.

De quels pouvoirs la musique (ou son écoute) serait-elle dépositaire pour devenir une cible privilégiée de la violence terroriste ? De quelle façon des pratiques musicales sontelles mobilisées, comme outil de propagande ou arme de guerre, par les mêmes personnes qui les attaquent ? L'objectif de cet article est de contribuer à esquisser une réponse à ces questions. Pour cela, j'analyserai les arguments mobilisés par l'idéologie salafiste et la propagande djihadiste afin de justifier l'interdiction de la musique - l'idée que la quasi-totalité de la musique serait haram (interdite). De même, j'examinerai les 
enjeux de la mobilisation des anasheed, un genre de chant polyphonique issu de la tradition musicale et poétique arabe, dans le mouvement djihadiste international ${ }^{17}$. Finalement, j'analyserai de quelle façon l'État islamique utilise le nasheed «Avance, avance » dans le communiqué audio de revendication des attentats de Paris, afin de ritualiser sa propagande, de légitimer la violence, d'encourager ses combattants et de démoraliser son ennemi.

Les sources étudiées seront principalement des vidéos, en français ou sous-titrées en français, mises en ligne sur YouTube ou sur d'autres réseaux sociaux. L'accessibilité de ces vidéos et leur rôle dans l'embrigadement des jeunes européens dans le djihad islamique ne doivent en aucun cas être sous-estimés ${ }^{18}$. Comme le remarque Farhad Khosrokhavar :

De nombreux individus en Occident qui souffrent de l'anomie et de la déstructuration des liens sociaux trouvent dans la Toile jihadiste une communauté d'autant plus attrayante qu'elle procure un sentiment intense d'appartenance, le sentiment que l'existence trouve un sens dans la lutte contre un ennemi perfide (l'Occident) et surtout, qu'en luttant contre cet Occident maléfique on expulse de son âme la part du diable qui s'y est faufilée. [...] L'internaute gagne une nouvelle identité en dénonçant sa propre part du diable. [...] La Toile permet d'opérer cette mutation identitaire dans un univers mi-onirique mi-réel qui fourmille de milliers de textes, de vidéos, de films et de témoignages, l'individu pouvant parcourir en quelques heures ce qui, dans le monde réel, prendrait des jours ou des mois ${ }^{19}$.

6 Le but de ce texte n'est pas de faire une exégèse du discours salafiste sur la musique, mais d'étudier la mobilisation de ces discours dans la propagande djihadiste à partir des documents accessibles en français aux jeunes susceptibles d'être embrigadés ou à toute personne qui se sentirait concernée par ces questions. Il s'agit de tenter de comprendre de quelle façon les enjeux de l'interdiction de la musique par l'idéologie salafiste, doublée de la propagande djihadiste de l'État islamique, peuvent contribuer à la radicalisation des personnes habitant en Europe en général, et en France et en Belgique en particulier. De même, il s'agit aussi de tenter d'expliciter le rôle joué par la musique dans un raisonnement qui rendrait possible la déshumanisation de l'« ennemi ", ceci étant l'une des conditions préalables à certains actes terroristes ou massacres de masse. Enfin, dans une perspective plus vaste, ce texte se donne pour objectif de contribuer à une meilleure compréhension des processus de mobilisation d'objets symboliques et des pratiques culturelles dans le dispositif de propagande djihadiste de l'État islamique.

\section{"La musique est haram » : les pratiques musicales et l'idéologie salafiste}

7 L'histoire de la censure de la musique ou des pratiques musicales est extrêmement vaste et ne se limite en aucun cas à l'islam. De la Grèce antique aux démocraties libérales, en passant par l'hindouisme, le christianisme et le judaïsme, on peut affirmer que toutes les religions et types de régimes politiques ont interdit ou censuré à un moment donné des œuvres ou des pratiques musicales. Dans de nombreux cas, cette interdiction se base sur le pouvoir d'influence qu'aurait la musique sur les émotions humaines ou sur le danger qu'elle pourrait représenter pour l'ordre social ${ }^{20}$. Concernant les rapports entre islam et musique, comme l'explique Amnon Shiloah, le terme $s a m a^{21}$ (qui signifie à la fois écoute musicale et musique) a donné lieu à une grande quantité d'écrits polémiques qui traitent sur l'autorisation ou l'interdiction de 
celle-ci « selon un point de vue juridique, théologique et mystique $»^{22}$. Ce débat suscite «des points de vue contradictoires: de l'interdiction absolue, d'une part, à l'autorisation de toutes les formes musicales, y compris la danse, d'autre part ${ }^{23}$. Cependant, de nombreux écrits nuancent ces deux positions. Par exemple, certains tolèrent «une forme rudimentaire de cantillation et des chants fonctionnels, mais bannissent les instruments». D'autres points de vue «autorisent la cantillation en y ajoutant le tambour sur cadre, du moins sans cymbalettes, mais réprouvent toute autre espèce d'instruments de musique et toutes formes de danse ${ }^{24}$.

8 Les discours actuels à propos de l'interdiction de la musique par l'idéologie salafiste tentent de puiser sa légitimé dans ces textes polémiques ${ }^{25}$. Toutefois, dans les vidéos diffusées en français et destinées tout particulièrement aux jeunes, elle prend la forme d'une critique des dérives de la société de consommation, qui seraient à la base du manque de perspectives d'avenir qu'offre cette société. Dans ces vidéos, sont dénoncées certaines de ces dérives, notamment les vices qu'encouragerait l'industrie du disque (qui produit notamment la variété, le rock, le rap ou le métal) et l'absence de spiritualité des sociétés occidentales contemporaines. La corruption de la société, à l'origine du mal-être de ses citoyens, serait le résultat des complots des dirigeants politiques, du "système », des "faux » musulmans ou des sociétés secrètes ${ }^{26}$ ainsi que du détournement des hommes et des femmes de $\operatorname{Dieu}^{27}$. Le rôle joué par la musique y serait très important. Pour certains imams qui prônent l'idéologie salafiste, il faudrait s'interroger sur l'influence des chansons et des « vidéos musicales, qui transforment les adorateurs ascètes en des libertins pervers, [...] où la femme se dandine comme un serpent et où l'homme se dandine comme la femme $»^{28}$. Changer ses habitudes et arrêter d'écouter de la musique est donc proposé comme une solution simple, et à la portée de tous, pour atteindre une paix intérieure qui permettrait de trouver un sens à sa vie tout en se rapprochant de l'islam « véritable ». Selon Farhad Khosrokhavar :

Le salafisme remplit une fonction "contre-anomique» fondamentale, puisqu'il apporte des solutions imaginaires simples aux problèmes réels et complexes de la modernité, et met fin au sentiment de solitude et de perte de repères dont souffraient ses adeptes. [...] Dans la conception salafiste, l'islam est présenté comme l'antidote au laxisme et à l'impuissance moderne à régir la vie sans qu'elle sombre dans le désespoir et le nihilisme ${ }^{29}$.

9 Ainsi, dans une vidéo intitulée "Pourquoi Allah a t'il interdit la music [sic]", la voix d'un jeune homme explique de façon extrêmement claire et pédagogique, durant plus de seize minutes, les raisons pour lesquelles la musique serait interdite par l'islam. Juxtaposant des images diverses ${ }^{30}$ qui renforcent ou illustrent le propos du discours, le narrateur affirme sans détour que « la musique est cent pour cent interdite, illicite : il est interdit d'en écouter", tout en soulignant que seules «les musiques avec des instruments sont interdites » et que «les chants avec des voix humaines uniquement sont autorisés ${ }^{31}$. Avant d'approfondir sur un ton familier son explication des raisons de cette interdiction, la voix interpelle la personne qui écoute en lui annonçant : « Vous allez à présent vous rendre compte à quel point la musique est dangereuse. Voici les dangers de la musique. » Puis, l'exposé commence :

Étiez-vous conscient que la musique est un objet de manipulation? Étiez-vous conscient que celle-ci vous affecte dans vos choix personnels? Nous allons vous expliquer un peu plus en détail. Déjà il faut savoir que la musique met l'être humain dans certains états. Par exemple, écouter une musique triste va, souvent, vous rendre triste. Une musique qui bouge va vous exciter. La musique, en fait, affecte votre état général. 
Suivent des exemples précis concernant des cas où la musique pourrait détruire ou mettre en danger la vie de quelqu'un: "Nous connaissons une personne qui nous appelait fréquemment et qui nous parlait de suicide. Et devinez quoi, à chaque fois nous entendions que cette personne écoutait en fond les musiques tristes du film Titanic.» De même, la voix off dévoile certaines des stratégies supposées d'utilisation de la musique à des fins de manipulation, faisant appel au sens commun de la personne qui regarde la vidéo. Alternant citations du Coran, études pseudo-scientifiques et exemples concrets tirés de la vie quotidienne, l'argumentation a pour but de convaincre le spectateur que « la musique peut avoir un réel pouvoir de manipulation si elle est bien utilisée et peut vraiment masquer la réalité dans beaucoup des cas ». Par exemple, les gens auraient besoin de la musique pour danser car celle-ci «va les affecter physiquement pour ne plus trop réfléchir et pouvoir plus ou moins se lâcher et être à l'aise ». D'après le narrateur, à cause de la musique : "Vous perdez votre morale, elle masque vraiment les choses et vous met dans une ambiance qui est fausse [...] La musique peut vous affecter réellement, un peu comme l'alcool ${ }^{32}$. »

De fait, la critique de la société de consommation est accompagnée d'une explication paranoïaque du manque de perspectives d'avenir pour les jeunes, la musique étant l'un des rouages d'un « système » mis en place pour maintenir l'ordre social, empêcher tout changement politique ou économique et détourner les individus de la «religion authentique $»^{33}$. Ce complot ferait en sorte que les gens "ne se posent plus des questions critiques et soient fondus dans le système, avec comme unique but : je fais la fête et je gagne de l'argent. Pas de réflexion. La technologie vous masque la vérité. La musique, les films, les jeux vous masquent la vérité». Ainsi, par sa capacité à nous influencer, la musique - et plus précisément son écoute - serait responsable du fait que nous nous détournons de la vérité délivrée par le Coran, elle nous empêcherait de voir clair et nous ferait accepter la société corrompue et injuste dans laquelle nous vivons. De même, l'emprise de la musique sur nous serait exacerbée par les pratiques d'écoute actuelles (nomades ou en ligne), qui seraient particulièrement nocives, car l'omniprésence de la musique dans notre vie quotidienne nous isolerait et rendrait impossible la réflexion :

De nos jours, les gens se lèvent et mettent la musique, l'écoutent avec le téléphone dans le bus, l'écoutent dans la voiture, les gens l'écoutent au travail. Énormément de gens se lèvent avec la musique, et se couchent avec la musique. Et finalement vivent une grande partie de leur vie avec un fond sonore. Comprenez à quel point celle-ci empêche de réfléchir convenablement et gêne le cerveau. Le meilleur moyen de réfléchir sérieusement à quelque chose c'est de n'avoir aucun son autour de vous car votre cerveau peut se concentrer vraiment. Sinon, celui-ci est constamment dérangé par ce qu'il entend.

12 La musique, ce bruit de fond dénoncé par l'idéologie salafiste, serait l'un des obstacles à l'écoute des paroles du Prophète. L'incompatibilité entre le "véritable » islam et la musique est donc mise en avant de façon systématique : «Si vous voulez vous dévouer sincèrement et complètement à l'islam, il ne faut pas que votre tête soit remplie de musique. Il y a des choses, mes frères et sœurs, qui ne peuvent pas vraiment coexister.» Des exemples de paroles de chansons de Marylin Manson («Antichrist Superstar») et du rappeur Eminem ("Say Goodbye Hollywood») ainsi que de supposées déclarations de John Lennon - «Je sais que les Beatles connaitront le succès comme aucun groupe ne l'a encore connu, [...] car pour ce succès j'ai vendu mon âme au diable» - viendraient confirmer l'idée du complot satanique de la musique et de 
l'industrie du disque ${ }^{34}$. Les personnes qui écoutent la musique seraient sous son emprise et leur cœur serait imprégné de « mauvaises choses» :

Vous savez mes frères et sœurs, le cœur est comme une éponge, il absorbe ce qu'il y a autour de lui. Donc notre cœur peut être noir et blanc selon ce que vous lui donnez. Vous voulez laver votre cœur, entourez lui [sic] des bonnes choses - du Coran, des prières, etc. Il l'absorbera et s'imprégnera de ça. Mais si vous écoutez et regardez constamment des choses obscènes, votre cœur s'imprégnera donc de ça et s'attachera à ces choses puisqu'il est complètement imbibé. Et vous aurez l'impression d'aimer les péchés, en vous disant que ce n'est pas si grave que ça ${ }^{35}$.

Ainsi, leur cœur étant corrompu, les personnes qui écoutent de la musique sont considérées comme intrinsèquement mauvaises, en opposition à la pureté qui caractériserait le groupe des croyants $^{36}$. Cette idée peut amener les individus embrigadés par la propagande salafiste à déshumaniser les personnes qui aiment la musique, d'autant plus que ce discours est légitimé par des imams, y compris en France. En effet, le très médiatique imam salafiste de la mosquée Sunna de Brest, Rachid Abou Houdeyfa, est filmé en 2013 devant un groupe d'enfants affirmant que les personnes qui aiment et qui écoutent la musique « écoutent le diable » et qu' "à partir du moment où c'est des chants et [qu']il y a des instruments de musique, c'est haram ${ }^{37}$. Il met en garde les enfants contre l'écoute de tous les genres de musique, dans n'importe quelle langue, affirmant que dans le cas contraire «Allah va les transformer en singes et en porcs ». Ensuite, il rend responsables les personnes qui veulent écouter de la musique de leur propre «bestialisation », en affirmant que « ceux qui aiment la musique ce sont ceux qui aimeraient bien quelque part être transformés en singes et en porcs ${ }^{38}$. Ce type de déclaration renforce l'interdiction concernant les chants accompagnés d'instruments de musique, mais crée aussi inévitablement un dégoût ou une répulsion envers les personnes qui les écoutent. Dans l'idéologie salafiste, la musique aurait donc un rôle important dans la construction identitaire d'un Autre impur, obscène, qui aimerait les péchés et qui aurait un cœur corrompu par la voix du diable.

Dans ses recherches sur les massacres de masse, Jacques Semelin fait remarquer que « la "bestialisation" de l'ennemi est en effet un indice très important du possible déclenchement de la violence contre lui ${ }^{39}$. L'animalisation de l'Autre, du fait de son appartenance réelle ou imaginaire à un groupe social, linguistique, ethnique ou religieux, constituerait une étape importante dans le processus de désignation d'un ennemi et de sa potentielle destruction ${ }^{40}$. Cette forme de déshumanisation permettrait donc d'instaurer la distance psychologique nécessaire pour déclencher la violence contre un groupe désigné par avance. Comme le souligne Semelin, "la guerre est d'ailleurs productrice de ce genre de métaphores entre les soldats qui ainsi se persuadent de ne pas tuer des hommes $»^{41}$.

Les propos de Rachid Abou Houdeyfa devant le parterre d'enfants ont été dénoncés par certains députés après avoir été relayés par la presse, notamment suite aux attentats du 13 novembre à Paris ${ }^{42}$. L'imam s'était déjà défendu des accusations qui le visaient lors d'un prêche, le 24 septembre 2015, filmé et mis en ligne par l'équipe de production de la mosquée (Alwassat). À cette occasion, il explique "aux intolérants complexés » que ces propos, prononcés dans un cadre religieux, portaient sur des "récits prophétiques » et qu'il faudrait les interpréter en tant que tels. Insistant sur la liberté de croire en ces récits, il affirme que les contester serait s'attaquer à l'islam :

Le messager d'Allah [...] a cité un récit prophétique où il rappelle qu'il y aura ici, avant la venue de la fin des temps, des transformations. Et parmi ces 
transformations, des gens qui seront transformés en porcs et en singes. Parmi ces personnes-là, certaines personnes, qui écoutent certains types de musique. Et bien, nous y croyons et je vous dis c'est le prophète qui l'a dit, ce n'est pas moi. Et tous les musulmans du monde entier reconnaissent cela et citent ces récits. Et bien, si les gens attaquent ces récits prophétiques, ils attaquent l'islam ${ }^{43}$.

Concernant sa responsabilité vis-à-vis des enfants, l'imam déclare qu'il n'a jamais dit "que si une personne écoutait de la musique on ne devait pas la respecter $»^{44}$. De même, il leur aurait fait remarquer : «Si tu es à l'école et tu dois écouter la musique tu n'as pas le choix et tu dois respecter : la mosquée c'est la mosquée, l'école c'est l'école». Enfin, il se défend d'avoir dit que «tout type de musique est interdit en islam » ${ }^{45}$. Ce dernier point constitue un artifice rhétorique car, comme il l'a expliqué aux enfants, seuls les chants sans accompagnement instrumental, nommés anasheed (pluriel de nasheed), seraient autorisés par le Coran.

\section{Anasheed et djihadisme international}

Ce discours sur la musique, d'une part, et sur les anasheed, d'autre part, est confirmé par les explications des imams salafistes ou wahhabites ${ }^{46}$, perçus comme des autorités en matière d'interprétation du Coran. Véritables stars médiatiques dans le monde arabe et musulman, certains de ces imams disposent d'une présence permanente sur des chaînes transnationales de télévision (satellitaire ou sur Internet) ${ }^{47}$. Ils sont appelés à s'exprimer sur des sujets extrêmement divers - religieux, politiques, économiques ou sociaux - lors des conférences ou des interventions publiques. Ainsi, une vidéo sur YouTube (sous-titrée en français) montre l'imam wahhabite Mohamed Al-Ari, expliquant sur la chaîne nationale qatarie (Qatar Télévision) :

À propos de la musique, il y a beaucoup de preuves qui montrent qu'elle est haram. Mais aujourd'hui, Al-Hamdoulillah [louange à Allah], nous n'avons plus besoin de musique, même dans les mariages, grâce aux anasheed. Dans les anasheed, les voix ont remplacé les instruments, qui sont haram, comme le piano ou la flûte. Vous n'avez plus besoin d'instruments de musique, puisqu'il y a aussi beaucoup de sons que vous pouvez faire grâce à votre ordinateur ${ }^{48}$.

De fait, la plupart des vidéos françaises qui abordent le sujet de l'interdiction de la musique dans l'islam sont accompagnées par des anasheed, avec ou sans paroles. Ces chants polyphoniques, hétérogènes sur le niveau de sophistication des plans sonores, sont systématiquement travaillés avec des logiciels audio rajoutant des effets divers (réverbération, écho, delay, filtres). Ceci dans le but, par exemple, de donner l'illusion que les chanteurs sont enregistrés dans de grands espaces (comme une mosquée), de renforcer l'importance d'un mot en le faisant se répéter plusieurs fois jusqu'à disparaître ou encore de donner plus de profondeur ou de présence aux voix en filtrant certaines fréquences. Dans tous les cas, le résultat recherché est une transformation de l'espace sonore ordinaire dans un espace situé ailleurs, "hors de la réalité ", qui donnerait aux chants une dimension envoûtante et magique. Comme le suggèrent les propos du Saoudien Mohamed Al-Ari, cette utilisation de la technologie ne semblerait pas poser de problème à l'idéologie salafiste, puisqu'il propose de supplanter les instruments par des sons de synthèse produits par l'ordinateur.

Au-delà de ce paradoxe, se trouve aussi l'idée que, sous certaines conditions, le chant pourrait ne pas rentrer dans la catégorie «musique ». Cette idée est avancée lorsque l'imam égyptien Mohamed Hassan affirme, sur la chaîne salafiste Al Rahma ${ }^{49}$ (« La 
Miséricorde »), que "le chant est une parole tant qu'il n'est pas accompagné d'instruments de divertissement et de musique $»^{50}$. Il serait donc possible de considérer qu'un chant ne relève pas de ce qu'on entend par "musique », mais du registre de la parole ou de la poésie, s'il n'est pas accompagné par des instruments de musique ${ }^{51}$. Ainsi, écouter des anasheed sans accompagnement ne serait pas pêcher : " Je conseille à mes sœurs et frères de ne pas écouter des anasheed lorsqu'ils sont accompagnés d'instruments de musique. En revanche, si ces anasheed ne sont pas accompagnés d'instruments, dans ce cas il n'y a aucun mal à les écouter ${ }^{52}$. " De même, le Saoudien Salih Al-Fawzan, membre du Conseil des grands oulémas d'Arabie Saoudite ${ }^{53}$, oppose dans une fatwa ${ }^{54}$ l'inshad al-shi'r (poésie récitée) aux anasheed soufis - qui relèveraient de la musique, n'appartiendraient pas au véritable islam et seraient néfastes pour l'étude du Coran ${ }^{55}$. L'idée que les anasheed relèvent de la poésie récitée, et non de la musique, remonte aux origines du genre, comme l'explique Amnon Shiloah :

La magie du rythme jointe au verbe conduisit à élaborer une poésie classique déclamée sous forme de récitation. On l'a appelée inshad, ce qui littéralement signifie " élever la voix » - anshada - et, partant, inshad al-shi'r (déclamation étirée de la poésie réalisée avec une voix sourde). Ce sens a donné un dérivé, nashid: terme qui, dans une période plus tardive, a désigné différentes formes musicales. S'appliquant également à l'origine au fait d'élever la voix, ce dernier terme s'est enrichi d'une connotation musicale : réciter la poésie en y mettant une certaine quantité de chant était déjà en pratique bien avant l'islam et s'est maintenu bien après. Chantée ou déclamée, la poésie représentait un symbole, un lien unificateur entre les populations nomade et sédentaire ${ }^{56}$.

Les vidéos de propagande salafiste en français ne définissent pas les anasheed en tant que " poésie récitée » mais comme la seule «musique » autorisée par l'islam. De même, elles n'abordent pas les paradoxes soulevés précédemment concernant l'utilisation de la technologie ou la méfiance partagée aussi bien par le salafisme que par le wahhabisme vis-à-vis des anasheed. Dans ses recherches sur les anasheed djihadistes, Behnam Said montre la difficulté des théoriciens salafistes et wahhabites à définir des critères précis pour justifier l'interdiction ou l'autorisation de ces chants, concluant que cette tradition des anasheed est plus proche de l'idéologie prônée par les Frères Musulmans, notamment depuis le mouvement de "renaissance islamique » des années 1970-198057. En effet, le théologien salafiste Nasir Al-Din Al-Albani considère que les anasheed islamiques sont autorisés seulement si :

a) [...] la mélodie n'est pas similaire à celle enseignée dans la musique occidentale et orientale, qui fait danser les gens.

b) [...] le texte est véritablement islamique.

c) [...] aucun instrument de musique n'est utilisé, à l'exception du duff (qu'il est permis de jouer seulement aux femmes durant les mariages).

d) [...] l'écoute des anasheed ne constitue pas une distraction de l'étude du Coran ${ }^{58}$.

Pour les wahhabites, les anasheed sont autorisés en tant qu'alternative à la musique populaire car ils suscitent, chez l'auditeur et le chanteur, "obéissance à Dieu et répandent un enthousiasme religieux qui repousse le diable $»^{59}$. Cependant, ils doivent être chantés seulement lors d'occasions spéciales (comme les mariages ou les voyages djihadistes) et leur écoute ne doit pas devenir une préoccupation constante ni écarter l'auditeur de l'étude du Coran. Abu Usama Ash-Shami émet une fatwa dans laquelle il affirme qu' il n'y a pas d'objection à l'écoute des anasheed s'ils ne comprennent pas de rythme, de musique, de percussions et d'influence [émotionnelle]. Ils sont autorisés notamment s'ils évoquent le courage [hamasa], comme les anasheed djihadistes $"{ }^{60}$. AlAlbani écrit à propos de cette catégorie d'anasheed: «Il y a les combattants qui 
chantent des poésies incitant à la bataille. Il y aussi le chant de ceux qui se préparent au combat chantant des poèmes pour se vanter au moment de la rencontre. [...] Ceci permet de faire bouger [...] les hommes sauf que cela n'implique pas d'extase faisant sortir du juste milieu ${ }^{61}$.»

Ces avis théologiques soulignent la dimension religieuse des anasheed et l'importance de leur rôle dans le djihad. Pour l'imam états-unien d'origine yéménite et idéologue d'Al-Qaïda Anwar Al-Awlaki, tué au Yémen par le missile d'un drone états-unien en 2011, les anasheed sont l'une des façons privilégiées de soutenir le djihad - «l'acte le plus grand dans l'islam " ${ }^{62}$. Dans son livre 44 Ways to Support Jihad (44 façons de soutenir le Djihad), il écrit :

\section{Les anasheed}

Les musulmans doivent être inspirés pour pratiquer le djihad. Au temps de Rasulullah (saaws), il y avait des poètes qui utilisaient leur poésie pour inspirer les musulmans et démoraliser les mécréants. Aujourd'hui le nasheed peut jouer ce rôle. Des anasheed de bonne qualité peuvent s'étendre si largement qu'ils peuvent atteindre un auditoire que vous ne pourriez pas atteindre par un cours ou un livre. Les anasheed sont particulièrement inspirants pour la jeunesse, qui est la base du djihad dans chaque époque. Les anasheed sont un élément important dans la création d'une " culture du djihad». Les anasheed sont nombreux en arabe, mais ils sont rares en anglais. Ainsi, il est important pour les poètes et les chanteurs doués de prendre cette responsabilité. Les anasheed peuvent couvrir des sujets comme : le Martyr, le djihad est notre seule solution, le soutien aux actuels leaders du djihad (pour le mettre en relation avec la jeunesse), la situation de l'oumma, la responsabilité de la jeunesse, la victoire de l'Islam et la défense de la religion. Les anasheed devraient se concentrer sur la justice plutôt que sur la paix, et sur la force plutôt que sur la faiblesse. Les anasheed doivent être forts et édifiants et non pas tristes et féminins ${ }^{63}$.

23 Les anasheed peuvent devenir les symboles collectifs d'une « communauté imaginaire » de djihadistes, remémorer les martyrs, construire une mémoire ou un imaginaire commun, réguler les émotions des combattants ou ritualiser la violence. Comme le montre Tia De Nora, la musique peut être considérée comme un «dispositif pour clarifier l'ordre social, pour structurer la subjectivité (le désir et les paramètres temporels des émotions, et la dimension émotive de l'interaction) ainsi que pour établir une base pour une action concertée $»^{64}$.

Ainsi que l'ont fait d'autres groupes armés djihadistes, tels qu'Al-Qaïda, Al-Qaïda au Maghreb islamique (AQMI) ou le Front Al-Nosra, l'État islamique utilise des anasheed dans certains de ses communiqués et ses vidéos de propagande ${ }^{65}$. Associés à ces vidéos, ils constituent un outil précieux pour réguler les émotions des spectateurs, aussi bien sur des images de batailles et de communiqués que dans la mise en scène d'exécutions d'ennemis du Califat. C'est pourquoi l'État islamique confère une importance stratégique à la maîtrise de sa propre production d'anasheed et prend grand soin de les diffuser à l'intérieur et à l'extérieur du territoire qu'il contrôle. Pour cela, le Califat s'est doté dès début 2014 de sa propre société de production, l'Ajnad Media Foundation, capable de produire un grand nombre d'anasheed djihadistes et de les diffuser sur Internet ${ }^{66}$. Il s'est doté aussi d'autres sociétés de production audiovisuelle, comme le AlFurqan Institute for Media Production ou le Al-Hayat Media Center, spécialisées dans la production de vidéos de propagande sophistiquées ou de revues multilingues en ligne ${ }^{67}$. 


\section{Les anasheed de l'État islamique : propagande et ritualisation de la violence}

En accord avec leur idéologie concernant l'influence de la musique sur les émotions des individus, l'objectif de l'État islamique est d'utiliser les anasheed comme une arme de guerre pour «jeter la crainte» dans le cœur de l'ennemi, pour persuader des combattants étrangers de les rejoindre, pour consolider une identité collective et motiver ses propres soldats, ou bien pour ritualiser et mettre en scène son pouvoir ${ }^{68}$. Le communiqué audio de revendication des attaques de Paris est constitué d'un discours parlé et d'un nasheed - aussi bien dans la version arabe du communiqué que dans la version française. La parole chantée et le discours parlé se disputent le premier et le deuxième plan du communiqué, le nasheed étant présent tout au long des cinq minutes et vingt-quatre secondes du fichier audio. La parole chantée - exemple de poésie djihadiste en langue française - est un chant de guerre polyphonique écrit à la deuxième personne du singulier qui introduit, ritualise et prolonge le contenu du communiqué69.

La voix du discours parlé du communiqué a été identifiée comme celle du djihadiste français Fabien Clain et la voix du nasheed comme celle de son frère cadet Jean-Michel Clain $^{70}$. Tout semble indiquer que les deux frères seraient aussi les auteurs des paroles du nasheed et se trouveraient actuellement en Syrie. Le projet de chanter des anasheed était déjà énoncé plusieurs années auparavant par Fabien Clain, lors du procès de sa filière djihadiste en 2008. Celui-ci aurait déclaré aux policiers : « Mon frère Jean-Michel et moi chantons ensemble, nous avons comme projet de former un groupe qui s'appellerait "rappeleur", des chants de rappel à l'islam, des nasheed [sic]. J'ai été rappeur avant d'être poète. Puis, après conversion à l'islam j'ai tout arrêté ${ }^{71}$. "

Le but du nasheed «Avance, avance » est bien de montrer la puissance du Califat, de terroriser l'ennemi et d'encourager les combattants de l'État islamique à poursuivre la

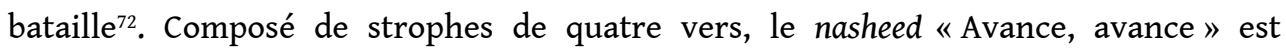
structuré en trois blocs, formés chacun d'un refrain écrit en rimes croisées (ABAB) et de deux couplets écrits en rimes suivies (AABB), le tout se terminant par un refrain final. Le nasheed est composé dans un langage tonal pour des voix masculines, dans une mesure à quatre temps et un tempo vif. Le contraste entre le refrain (huit mesures) et les couplets (huit mesures par couplet) est assuré par une opposition sur la texture et l'écriture des voix (homophonie/polyphonie), d'une part, et par une opposition sur le plan harmonique (stable/instable), d'autre part. Ainsi, la mélodie répétitive et syncopée du refrain est caractérisée par une écriture homophonique et une harmonie relativement statique. En revanche, le couplet est résolument polyphonique (à trois ou quatre voix), utilisant des procédés contrapuntiques variés (tuilages, superposition d' ostinati, bourdons, imitations) et instable harmoniquement ${ }^{73}$. Enfin, les paroles du nasheed mobilisent des images d'une violence extrême ${ }^{74}$, des exaltations à exterminer l'ennemi et des symboles issus de la poésie arabe classique (le lion, par exemple) :

\footnotetext{
"Avance, avance "

Avance, avance, avance, avance,

Sans jamais reculer, jamais capituler,

Avance, avance, avance, avance,

Guerrier invaincu, l'épée à la main tue-les !

Tue les soldats du diable sans hésitation

Fais-les saigner même dans leur habitation
} 
N'aie peur de rien, fonce tout droit vers le bonheur Le champ de la bataille est le champ des honneurs. Dans cette guerre tu as tout à y gagner Un beau jour ta sueur et ton sang vont témoigner Bats-toi jusqu'à la rencontre du Tout puissant En courant vers ta proie, tu es là, Lion rugissant !

Avance, avance, avance, avance,

Sans jamais reculer, jamais capituler, Avance, avance, avance, avance, Guerrier invaincu, l'épée à la main tue-les ! Tue les apostats que le diable a égarés Avec les gens du Fou la guerre est déclarée Plus de polémique ni de philosophie Sois tu les tues, soit ils te tuent, que du profit. Quiconque s'oppose à la charia est perdu Même lorsqu'il prétend pratiquer la vertu Alors coupe les têtes de l'ignorance Coupe les têtes des soldats de l'errance! Avance, avance, avance, avance, Sans jamais reculer, jamais capituler, Avance, avance, avance, avance, Guerrier invaincu, l'épée à la main tue-les ! Tue les traîtres, attaque-les par surprise Égorge-les, fais leur payer leur traitrise Identifie l'hypocrite au cœur mort qui bat Qui ne bat que pour les intérêts d'ici-bas. Il pense qu'Allah ne va pas le dévoiler Quel vrai imbécile, inconscient au cœur voilé Achève-le d'une balle dans la tête

Tel est le sort du criminel qui s'entête !

Avance, avance, avance, avance, Sans jamais reculer, jamais capituler, Avance, avance, avance, avance, Guerrier invaincu, l'épée à la main tue-les. Avance !

L'analyse du sonagramme du fichier audio du communiqué nous montre un équilibre soigné dans la forme générale entre la voix chantée et la voix parlée (Nasheed - Discours - Nasheed), chacune occupant exactement la moitié du temps du communiqué (voir exemple 1). Après l'exposition du refrain d'« Avance, avance » comme introduction (15 secondes), vient le discours parlé sur fond de nasheed ( 2 minutes 42 secondes), puis le nasheed complet ( 2 minutes 27 secondes) ${ }^{75}$. L'équilibre de cette forme requiert un savoir-faire certain et montre à quel point l'État islamique réfléchit aux dimensions esthétique et rituelle de sa propagande, donnant une importance particulière aux détails. Le refrain du nasheed ouvre le cadre rituel, soulignant le caractère "sacré » de la parole qui le suit, puis le nasheed de fin prolonge le discours parlé et apporte une dimension collective au rituel grâce à son caractère polyphonique.

Mais le sonagramme dévoile aussi deux sources sonores avec des spectres de fréquences hétérogènes, donnant des informations importantes concernant la façon dont le communiqué a été fabriqué. La coupure brutale du spectre du nasheed autour de $14,6 \mathrm{kHz}$ prouve que le fichier utilisé dans le mix final du communiqué est un fichier compressé en format mp3 à $128 \mathrm{kbps}$ (nombre des kilobits par seconde). Ce fait montre que les deux sources n'ont pas été enregistrées au même endroit ni avec le même 
matériel (hardware) car elles auraient un spectre similaire (allant jusqu'à $20 \mathrm{kHz}$, comme c'est le cas dans la version arabe du communiqué). Dans le cas contraire il n'y aurait aucune raison de compresser l'un des deux fichiers et de réduire considérablement ses qualités sonores. Il est possible d'en déduire que le nasheed a été enregistré ailleurs et qu'il a été compressé pour des raisons de " portabilité ", afin qu'il soit plus léger et ainsi pouvoir l'envoyer par internet utilisant une messagerie électronique standard avec une connexion bas débit ${ }^{76}$. Si la voix appartient bien à Fabien Clain, qui vraisemblablement se trouvait en Syrie au moment des attentats, cette hypothèse permet de supposer que le nasheed « Avance, avance » a été enregistré par Jean-Michel Clain dans un autre pays, puis envoyé en Syrie afin d'être monté dans le communiqué de revendication. Cette hypothèse permet de penser le dispositif de propagande numérique de l'État islamique non seulement comme un conglomérat de ressources techniques, matérielles et humaines qui se trouvent sur le territoire de Califat, mais aussi comme un réseau global, une constellation potentielle de ressources situées partout dans le monde.

Exemple. Sonagramme du communiqué de revendication de l'État islamique (14 novembre 2015). Le spectre des fréquences des deux sources est clairement différencié

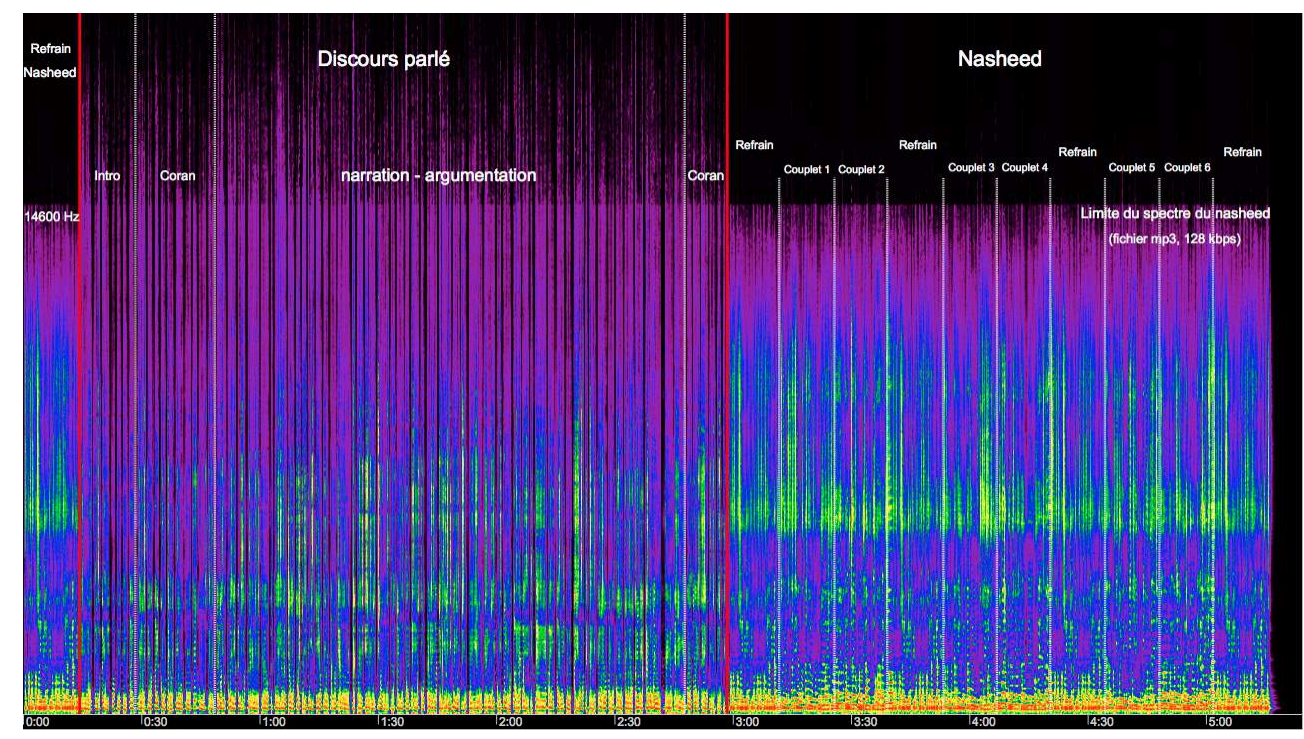

Sonagramme réalisé avec le logiciel d'analyse EAnalysis (http://logiciels.pierrecouprie.fr/? page_id=402)

L'utilisation de ce nasheed permet aussi de créer une chaîne d'équivalence symbolique entre les attentats de Paris et d'autres actes de guerre de l'État islamique, comme l'exécution du sous-lieutenant Maaz Al-Kassasbeh, pilote de chasse de la Force aérienne royale jordanienne, brûlé vif au début de l'année 2015. En effet, le nasheed utilisé dans le communiqué de revendication des attentats de Paris est une version en français du nasheed composé pour la vidéo qui justifie et met en scène le châtiment par le feu du pilote - en représailles aux bombardements de la Jordanie et de la coalition internationale ${ }^{77}$. Bien avant les attentats de Paris, les poètes-chanteurs de l'État islamique avaient écrit des paroles en français, qui conservent le sens général de la version arabe mais sans constituer une traduction littérale ${ }^{78}$. La mélodie et la forme du nasheed sont adaptées à ce nouveau texte. La réutilisation du nasheed permet au Califat 
de se référer implicitement à la justice appliquée au pilote en tant que projet politique et d'amplifier les émotions causées par les deux événements afin de terrifier l'ennemi.

Dans cette vidéo de propagande d'une grande sophistication ${ }^{79}$, produite par Al-Furqan Institute for Media Production et intitulée "Healing the believers' chests ${ }^{80}$, le nasheed apparaît dans l'introduction de la vidéo, où est montré le rôle de la Jordanie dans la coalition internationale contre le Califat. Puis, le pilote est filmé faisant son autocritique et expliquant en détail l'opération militaire et les bombardements aériens dans lesquels il est impliqué. Dans un montage qui fait s'alterner des plans sonores et visuels contrastés, des cadavres de victimes (pour la plupart des enfants) brûlées ou ensevelies sont montrés afin de justifier son châtiment. Ensuite, vient la mise en scène de son exécution (la ritualisation de sa mise à mort), où il sera brûlé vif à l'intérieur d'une cage métallique, puis enseveli sous un tas de décombres à l'endroit supposé d'un de ses bombardements ${ }^{81}$. Le nasheed apparaît graduellement en fondu d'ouverture (fadein), après une longue séquence de silence, au moment où le feu s'approche de la cage métallique et embrase le pilote, provoquant un effet dramatique insoutenable. La parole chantée continue jusqu'à la fin de la vidéo, où l'État islamique fait apparaître des portraits d'autres "pilotes croisés" jordaniens mobilisés dans les opérations de la coalition (avec nom, adresse et détails personnels), offrant une récompense pour la mort de chacun d'entre eux.

Le nasheed ritualise l'introduction du récit puis la mise à mort de l'ennemi dans la vidéo, d'une part, et le discours parlé dans le communiqué, d'autre part. La forme générale est la même (Nasheed [introduction] - Discours [justification] - Nasheed [mise à mort]) que dans d'autres communiqués de revendication ou vidéos d'exécutions ${ }^{82}$, montrant la volonté de l'État islamique de créer un lien symbolique entre ceux-ci en instaurant une sorte de liturgie politique de la mise à mort. Cette liturgie mobilise des émotions et nourrit un imaginaire commun, contribuant à consolider un récit des "victoires» et de la justice de l'État islamique tout en construisant une mémoire transnationale du Califat. Comme le remarque Philippe-Joseph Salazar, il est frappant de constater «à quel point le Califat suit des rituels extrêmement organisés. Ils ont quasiment une vision liturgique de la mise à mort. [...] C'est dans la duplication des rituels que le message acquiert de plus en plus de lourdeur $»^{83}$. Le nasheed produit un lien symbolique entre le châtiment subi par les ennemis lors des actes de guerre, les ancrant dans une histoire et un projet politique, tout en incitant les combattants djihadistes à prolonger le combat ailleurs dans le monde. L'efficacité du dispositif est redoutable.

\section{Conclusion}

Les pratiques musicales occupent une place centrale dans le processus de construction d'un collectif recherché par l'État islamique. Les enjeux de l'interdiction et de l'utilisation de la musique dans la propagande djihadiste révèlent de façon édifiante le projet idéologique de purification interne et d'extermination externe de l'organisation terroriste. La musique, ou plutôt l'écoute et les pratiques musicales, sont dépositaires d'attributs de pureté-impureté qui essentialisent une identité collective. L'interdiction de l'écoute de la musique constitue un marqueur identitaire non négociable entre les personnes qui seraient à l'intérieur et à l'extérieur du groupe. Elle isole aussi le jeune embrigadé de son habitat acoustique quotidien - cette «fonderie où les différentes 
émotions se trouvent identifiées, articulées, expérimentées et travaillées en temps réel $»^{84}$ - en lui substituant à ce dernier un nouvel environnement ${ }^{85}$. La dimension affective mobilisée par l'écoute des anasheed djihadistes joue ainsi un rôle clé dans la nouvelle construction identitaire, structurant et ritualisant les discours et les pratiques de socialisation. Comme le remarque Chantal Mouffe :

C'est toujours par leur insertion dans de multiples pratiques, discours et jeux de langage que se construisent les formes spécifiques d'identité. C'est pourquoi la transformation des identités politiques ne peut jamais résulter d'un appel rationaliste au véritable intérêt du sujet, mais plutôt de l'inscription de l'agent social dans un ensemble de pratiques qui mobiliseront ses affects de façon à désarticuler le cadre où a lieu le processus dominant d'identification ${ }^{86}$. salafistes sur leur pouvoir à atteindre et à transformer le cœur des personnes. Paradoxalement, elle s'appuie sur le même dispositif idéologique que celui mobilisé pour interdire la musique et son écoute. De même, cette efficacité repose sur leur capacité à esthétiser, conjointement avec les discours et les images des vidéos, le projet politique du Califat ainsi que la violence comme moyen de le faire advenir. Concernant la vaste production des anasheed, « leur force persuasive d'étrangeté et de rupture est séduisante: elle ouvre sur un autre univers, qui semble être hors répétition, hors banalité, hors quotidien. Elle réenchante le monde. Elle lui donne du sens et permet de passer de l'attirance esthétique à l'adhésion éthique ${ }^{87}$.

La mobilisation de la musique dans le contexte de la guerre asymétrique contre l'État islamique n'est pas un phénomène marginal ou dérisoire. Après les attaques terroristes de Paris, La Marseillaise a été chantée lors de nombreuses et très diverses occasions (partout dans le monde), montrant sa capacité à être mobilisée, appropriée ou resignifiée en tant qu'hymne national, chant de rassemblement, de guerre, de deuil, d'espoir ou de mémoire. Des centaines de milliers des personnes l'ont chantée de façon spontanée - au Stade de France après l'attentat manqué, par exemple - ou préméditée et organisée lors de cérémonies officielles ${ }^{88}$, de matchs de football ${ }^{89}$ ou de concerts ${ }^{90}$. Pour sa part, l'État islamique semble avoir trouvé un chant de guerre pour symboliser son combat contre la France "croisée ». Le nasheed "Avance, avance », devenu cet hymne de guerre, est utilisé à nouveau dans une vidéo de propagande diffusée sur Internet le 20 novembre 2015, intitulée "Paris s'est effondré »" Il réapparaitra sans doute dans d'autres vidéos et d'autres communiqués.

Le dispositif de propagande de l'État islamique persuade et incite à l'action par la mobilisation de discours et d'objets symboliques dans des espaces aussi bien virtuels que réels. Il est d'autant plus efficace que la propagande mobilisée est très peu analysée et débattue par les médias. De fait, une des erreurs essentielles des hommes politiques, des journalistes, des experts et des intellectuels médiatiques est d'avoir refusé «d'envisager le djihadisme autrement que comme une pathologie d'imbéciles ${ }^{92}$. En «barbarisant » les combattants de l'État islamique, en les traitant de « décervelés » ou de "sauvages", ils se sont ôté la possibilité d'analyser et de réfléchir aux idées et aux dispositifs déployés par leur propagande, tout en la renforçant. L'analyse des enjeux de la musique au sein de cette propagande permet un accès privilégié à la mécanique idéologique de l'État islamique, soulignant l'importance des émotions dans les rituels et les processus d'identification mis en œuvre par cette organisation djihadiste. 


\section{BIBLIOGRAPHIE}

\section{Sources en Internet}

« Anasheed Halal ou Haram, Mohammed Hassan », https://www.youtube.com/watch? v=vAofyBu07AI (mis en ligne le 22 février 2014, consulté le 18 novembre 2015).

«Angleterre-France : Wembley chante en chœur la "Marseillaise" en hommage aux victimes des attentats », Francetvinfo, 17 novembre 2015, http://www.francetvinfo.fr/faits-divers/ terrorisme/attaques-du-13-novembre-a-paris/video-anglettere-france-wembley-chante-enchoeur-la-marseillaise-en-hommage-aux-victimes-des-attentats_1180347.html (consulté le 10 décembre 2015).

« Attaques à Paris : Le Bataclan "un cible [sic] par excellence, la musique complètement haram" pour les isl », TF1, 14 novembre 2015 (http://lci.tf1.fr/france/attaques-a-paris-le-bataclan-uncible-par-excellence-la-musique-8683502.html [consulté le 25 novembre 2015]).

"Congrès à Versailles : "La Marseillaise" chantée par les parlementaires après les discours de Hollande », Francetvinfo, 16 novembre 2015, http://www.francetvinfo.fr/faits-divers/ terrorisme/attaques-du-13-novembre-a-paris/video-la-marseillaise-entonnee-au-congres-aversailles_1178631.html (consulté le 10 décembre 2015).

« Déclaration à l'issue du Conseil de défense », Élysée, samedi 14 novembre 2015 (http:// www.elysee.fr/declarations/article/declaration-a-l-issue-du-conseil-de-defense-2 [consulté le 18 novembre 2015]).

« Eagles of Death Metal s'exprime sur les attentats de Paris », Vice news (https://news.vice.com/ $\mathrm{fr} /$ video/eagles-of-death-metal-s-exprime-sur-les-attentats-de-paris [consulté le 27 novembre 2015]).

«Écouter la musique encourt le risque d'être transformé en singe ou porc !!! », https:// www.youtube.com/watch?v=5wpBb7Ulqoc (mis en ligne le 21 septembre 2015, consulté le 18 novembre 2015).

«Écoutez ce frère svp / Islam musique haram », https://www.youtube.com/watch? v=VXioCk2IPGQ (mise en ligne le 14 mars 2012, consulté le 24 novembre 2015).

« \#EnMémoire », Le Monde (http://www.lemonde.fr/attaques-a-paris/visuel/2015/11/25/ enmemoire_4817200_4809495.html (consulté le 12 décembre 2015).

État islamique, «Communiqué sur l'attaque bénie de Paris contre la France croisée », https:// ia801505.us.archive.org/32/items/CommuniqueAudio/communique\%20audio.mp3 (mis en ligné le 14 novembre 2015, consulté le 15 novembre 2015). Version arabe du communiqué disponible ici : https://ia801508.us.archive.org/10/items/FranceArabic/FranceArabic.mp3 (mis en ligne le 14 novembre 2015, consulté le 8 décembre 2015).

État islamique, " Healing the believers' chests ", https://archive.org/details/ AlFurqaanMediaHealingTheBelieversChests (mis en ligne le 15 février 2015, consulté le 8 décembre 2015).

État islamique, « Killing the Apostates in Revenge For the Monotheists \#1 - Wilayat Khurasan », http://jihadology.net/2015/06/18/new-video-message-from-the-islamic-state-killing-theapostates-in-revenge-for-the-monotheists-1-wilayat-khurasan (mis en ligne le 18 juin 2015, consulté le 8 décembre 2015). 
État islamique, « Killing the Apostates in Revenge For the Monotheists \#2 - Wilayat Khurasan ", http://jihadology.net/2015/08/09/new-video-message-from-the-islamic-state-killing-theapostates-in-revenge-for-the-monotheists-2-wilayat-khurasan (mis en ligne le 9 août 2015, consulté le 8 décembre 2015).

État islamique, « Killing the Apostates in Revenge For the Monotheists \#3 - Wilayat Khurasan », http://jihadology.net/2015/12/07/new-video-message-from-the-islamic-state-killing-theapostates-in-revenge-for-the-monotheists-3-wilayat-khurasan (mis en ligne le 7 décembre 2015, consulté le 8 décembre 2015).

État islamique, « Paris s'est effondré », http://jihadology.net/2015/11/20/new-video-messagefrom-the-islamic-state-paris-has-collapsed-wilayat-\%E1\%B8\%A5alab (mis en ligne le 20 novembre 2015, consulté le 29 novembre 2015).

État islamique, «Soon, soon », https://archive.org/details/SoonSoon (mis en ligne le 9 décembre 2015, consulté le 12 décembre 2015).

« Islam - La Musique - La voix de Shaytan (du diable) par Rachid Abou Houdeyfa », https:// www.youtube.com/watch?v=VaMEdzdaw8M (mis en ligne le 22 janvier 2012, consulté le 24 novembre 2015).

« La Marseillaise retentit à Notre-Dame-de-Paris (15 novembre 2015)», https:// www.youtube.com/watch?v=Pa3YOKk-uLk (mis en ligne le 15 novembre 2015, consulté le 10 décembre 2015).

« La musique est haram ! », https://www.youtube.com/watch?v=tqqeQpGL7lo (mis en ligne le 3 septembre 2012, consulté le 18 novembre 2015).

« La musique est Haram (illicite) en Islam », https://www.youtube.com/watch?v=FGoriapzFrE (mis en ligne le 3 septembre 2012, consulté le 18 novembre 2015).

« La musique est strictement interdit [sic] en Islam! ", https://www.youtube.com/watch? $\mathrm{v}=\mathrm{cFMzAk5zGzo}$ (mis en ligne le 6 juillet 2012, consulté le 24 novembre 2015).

"L'imam R. Abou Houdeyfa sur la musique, les femmes... », https://www.youtube.com/watch? V=cZG-7G1DvVA (mis en ligne le 24 septembre 2015, consulté le 24 novembre 2015).

« Madonna - La Marseillaise - Live @ Paris (Bercy), 9/12/2015», https://www.youtube.com/ watch?v=AhwyG9XTjls (mis en ligne le 9 décembre 2015, consulté le 10 décembre 2015).

«Musique au temps de Saint Louis. Chanson de croisade », https://www.youtube.com/watch? $\mathrm{V}=\mathrm{oTUvdemWYzE}$ (mis en ligne le 2 mai 2014, consulté le 10 décembre 2015).

« Pourquoi Allah a t'il interdit la music [sic]», https://www.youtube.com/watch?v=uyFWfRuMVA (mis en ligne le 16 novembre 2014, consulté le 18 novembre 2015).

" The French hymn resounds to the Metropolitan Opera de NYC », https://www.youtube.com/ watch?v=16vGq_rHAmE (mis en ligne le 15 novembre 2015, consulté le 10 décembre 2015).

\section{Radio et presse}

BUI, Doan, « De Toulouse à la Syrie : Fabien Clain, le parrain djihadiste, qui a revendiqué les attentats ", L'Obs, 19 novembre 2015 (http://tempsreel.nouvelobs.com/attentats-terroristes-aparis/20151118.OBS9734/de-toulouse-a-la-syrie-fabien-clain-le-parrain-djihadiste.html [consulté le 28 novembre 2015]).

« Burning of the Murtadd Pilot », Dabiq, n 7, février 2015, p. 5-8. 
GUERIN, Michel, « Après le Bataclan, tous au spectacle ! », http://www.lemonde.fr/idees/article/ 2015/11/20/ils-ont-les-armes-on-a-les-pieces-montees_4814224_3232.html (consulté le 18 novembre 2015).

HABERMAS, Jürgen, « Le djihadisme, une forme moderne de réaction au déracinement », Le Monde, 23 novembre 2015, p. 18-19 (http://www.lemonde.fr/idees/article/2015/11/21/jurgenhabermas-le-djihadisme-une-forme-moderne-de-reaction-au-deracinement_4814921_3232.html? xtmc=habermas\&xtcr=1 [consulté le 10 décembre 2015).

KRISTANADJAJA, Gurvan, «L'imam de Brest, un "salafiste YouTube” au rôle ambigu », Libération, 23 novembre 2015 (http://www.liberation.fr/france/2015/11/23/l-imam-de-brest-un-salafisteyoutube-au-role-ambigu_1415599 [consulté le 10 décembre 2015]).

MORTAIGNE, Véronique et GUIBERT, Nathalie, « Le Bataclan, un haut lieu de la culture ciblé de longue date par les islamistes ", Le Monde, 16 novembre 2015 (http://www.lemonde.fr/attaquesa-paris/article/2015/11/15/le-bataclan-un-haut-lieu-de-la-culture-cible-de-longue-date-par-lesislamistes_4810424_4809495.html [consulté le 25 novembre 2015]).

MOULINE, Nabil, « Genèse du djihadisme », Le Monde diplomatique, $\mathrm{n}^{\circ}$ 741, décembre 2015.

«Que nous veut Daesh?», Culturesmonde, France Culture, 16 novembre 2015 (http:// www.franceculture.fr/emission-culturesmonde-que-nous-veut-daech-2015-11-16 [consulté le 18 novembre 2015]).

SEELOW, Soren et GUIBERT, Nathalie, « Fabien Clain, la "voix" du massacre de Paris, avait déjà menacé le Bataclan en 2009 », Le Monde, 18 novembre 2015 (http://www.lemonde.fr/attaques-aparis/article/2015/11/18/fabien-clain-la-voix-du-massacre-de-paris-avait-deja-menace-lebataclan-en-2009_4812298_4809495.html?xtmc=voix_communique_attentats\&xtcr=14 [consulté le 28 novembre 2015]).

Six jeunes fiers et en colère, « Message de notre jeunesse aux terroristes », Marianne, 14 novembre 2015, http://www.marianne.net/agora-message-notre-jeunesse-auxterroristes-100238057.html (consulté le 18 novembre 2015).

STIEGLER, Bernard, «Ce n'est qu'en projetant un véritable avenir qu'on pourra combattre Daech », Le Monde, 19 novembre 2015 (http://www.lemonde.fr/emploi/article/2015/11/19/ bernard-stiegler-ce-n-est-qu-en-projetant-un-veritable-avenir-qu-on-pourra-combattredaech_4813660_1698637.html?xtmc=declaration\&xtcr=13 [consulté le 10 décembre]).

\section{Ouvrages et articles}

ADRAOUI, Mohamed-Ali, Du Golfe aux banlieues, le salafisme mondialisé, Paris, Puf, 2013.

AL-ALBANI, Nasir Al-Din, Tahrim Alat at-Tarab, Maktaba Al-Dalil, 1996 [traduction française abrégée : La musique en Islam. Chants soufis, Anashid islamiques et les instruments de musique, Bruxelles, Al-Hadith, 2013].

AL-AWLAKI, Anwar, 44 Ways to Support Jihad, Victorious Media, [s.d.].

BÉNICHOU, David, KHOSROKHAVAR, Farhad, MIGAUX, Philippe, Le Jihadisme. Le comprendre pour mieux le combattre, Paris, Plon, 2015.

BOUZAR, Dounia, CAUPENNE, Christophe, VALSAN, Sulayman, « La métamorphose opérée chez le jeune par les nouveaux discours terroristes », CPDSI (http://www.bouzar-expertises.fr/images/ docs/METAMORPHOSE.pdf [consulté le 23 novembre 2015]).

BOUZAR, Dounia, Comment sortir de l'emprise « djihâdiste » ?, Ivry-sur-Seine, Éditons de l'Atelier, 2015. 
DE MONTALEMBERT, Eugène et ABROMONT, Claude, Guide des genres de la musique occidentale, Paris, Fayard/Henry Lemoine, 2010.

DE NORA, Tia, Music in everyday life, Cambridge, Cambridge University Press, 2000.

DE NORA, Tia, « Quand la musique de fond entre en action », Terrain, $\mathrm{n}^{\circ}$ 37, 2001 (http:// terrain.revues.org/1310).

HUSSEIN, Hasna, « La télévision et la transformation de l'éthique islamiste : les présentatrices niqabées sur les chaînes salafistes satellitaires arabes ", Recherches féministes, vol. 28, $\mathrm{n}^{\circ}$ 2, 2015, p. 223-242.

KHOSROKHAVAR, Farhad, Radicalisation, Paris, Éditions de la Maison des Sciences de l'Homme, 2014.

KORPE, Marie (éd.), Shoot the Singer! Music Censorship Today, Londres, Zed Books, 2004.

KORPE, Marie, REITOV, Ole et CLOONAN, Martin, « Music Censorship From Plato to the Present », in BROWN, Steven et VOLGSTEN, Ilrik (eds.), Music and Manipulation. On the Social Uses and Social Control of Music, New York/Oxford, Berghahn Books, 2006, p. 239-263.

LEMESTRE, Denis, Le Chamane et son chant. Relations ethnographiques d'une expérience parmi les Huicholes du Mexique, Paris, L'Harmattan, 2003.

MORGAN, Andy, Music, Culture and Conflict in Mali, Copenhague, Freemusic, 2013.

MOUFFE, Chantal, Agonistique. Penser politiquement le monde, Paris, Beaux-Arts de Paris éditions, 2014.

MOULINE, Nabil, Les Clercs de l'islam. Autorité religieuse et pouvoir politique en Arabie Saoudite (XVIII XXI ${ }^{e}$ siècle), Paris, Puf, 2011.

NAJI, Abu Bakr, The Management of Savagery. The Most Critical Stage Through Which the Umma Will Pass [2004], trad. W. Mc Cants, Cambridge (MA), University of Harvard, 2006.

PIESLAK, Jonathan, Sound Targets. American Soldiers and Music in the Iraq War, Bloomington, Indiana University Press, 2009.

PIESLAK, Jonathan, Radicalism and Music, Middletown, Wesleyan University Press, 2015.

SAID, Behnam, « Hymns (Nasheeds): A Contribution to the Study of the Jihadist Culture ", Studies in conflict and Terrorism, vol. 35, $\mathrm{n}^{\circ}$ 12, 2012, p. 863-879.

SALAZAR, Philippe-Joseph, Paroles armées. Comprendre et combattre la propagande terroriste, Paris, Lemieux Éditeur, 2015.

SEMELIN, Jacques, Purifier et détruire. Usages politiques des massacres et génocides, Paris, Éd. du Seuil, 2005.

SHILOAH, Amnon, La Musique dans le monde de l'islam, Paris, Fayard, 2002.

SHILOAH, Amnon, "Judaïsme et islam : les monothéismes face à la musique ", in NATTIEZ, JeanJacques (ed.), Musiques. Une encyclopédie pour le XXI ${ }^{e}$ siècle, vol. 3 « Musiques et cultures », Arles, Actes Sud/Cité de la Musique, 2005, p. 358-385.

STERNE, Jonathan, « The mp3 as cultural artifact », New Media and Society, vol. 8, n 5, 2006, p. 825-842.

VELASCO-PUFLEAU, Luis, " Reflections on Music and Propaganda », Contemporary Aesthetics, vol. 12, 2014, (http://quod.lib.umich.edu/c/ca/7523862.0012.007?view=text;rgn=main). 
WIKTOROWICZ, Quintin, « Anatomy of the Salafi Movement », Studies in Conflict and Terrorism, vol. 29, n 3, 2006, p. 207-239.

\section{NOTES}

1. Concert du groupe états-unien Eagles of Death Metal.

2. Des communiqués écrits en plusieurs langues ont été diffusés simultanément au communiqué audio en arabe et en français.

3. «Communiqué sur l'attaque bénie de Paris contre la France croisée » (extrait), diffusé sur Internet le 14 novembre 2015 (https://ia801505.us.archive.org/32/items/CommuniqueAudio/ communique\%20audio.mp3 [consulté le 15 novembre 2015]). La version arabe du communiqué est disponible ici: https://ia801508.us.archive.org/10/items/FranceArabic/FranceArabic.mp3 (consulté le 8 décembre 2015).

4. NAJI, Abu Bakr, The Management of Savagery. The Most Critical Stage Through Which the Umma Will Pass [2004], trad. W. Mc Cants, Cambridge (MA), University of Harvard, 2006, p. 32. Sorte de manuel de stratégie, cet ouvrage théorise pas à pas les étapes pour constituer le Califat et reste une des principales sources idéologiques de l'État islamique.

5. Daesh est l'acronyme arabe d'État islamique en Irak et au Levant, le nom de l'État islamique jusqu'à la proclamation du Califat, le 29 juin 2014, dans les territoires qu'il occupe. Pour le nommer, j'utiliserai les termes État islamique (afin de me référer à l'organisation politique et militaire) ou Califat (afin de me référer à leur territoire ou à leur régime politique).

6. "Déclaration à l'issue du Conseil de défense ", Élysée, samedi 14 novembre 2015 (http:// www.elysee.fr/declarations/article/declaration-a-l-issue-du-conseil-de-defense-2 [consulté le 18 novembre 2015]).

7. «Que nous veut Daesh?», Culturesmonde, France Culture, 16 novembre 2015 (http:// www.franceculture.fr/emission-culturesmonde-que-nous-veut-daech-2015-11-16, consulté le 18 novembre 2015).

8. Voir, par exemple, Six jeunes fiers et en colère, " Message de notre jeunesse aux terroristes ", Marianne, 14 novembre 2015, http://www.marianne.net/agora-message-notre-jeunesse-auxterroristes-100238057.html (consulté le 18 novembre 2015).

9. GUERIN, Michel, «Après le Bataclan, tous au spectacle!», http://www.lemonde.fr/idees/ article/2015/11/20/ils-ont-les-armes-on-a-les-pieces-montees_4814224_3232.html (consulté le 18 novembre 2015).

10. Voir STIEGLER, Bernard, "Ce n'est qu'en projetant un véritable avenir qu'on pourra combattre Daech", Le Monde, 19 novembre 2015 (http://www.lemonde.fr/emploi/article/ 2015/11/19/bernard-stiegler-ce-n-est-qu-en-projetant-un-veritable-avenir-qu-on-pourra-

combattre-daech_4813660_1698637.html?xtmc=declaration\&xtcr=13 [consulté le 10 décembre 2015]) ; HABERMAS, Jürgen, " Le djihadisme, une forme moderne de réaction au déracinement ", Le Monde, 23 novembre 2015, p. 18-19 (http://www.lemonde.fr/idees/article/2015/11/21/jurgenhabermas-le-djihadisme-une-forme-moderne-de-reaction-au-deracinement_4814921_3232.html? xtmc=habermas\&xtcr=1 [consulté le 10 décembre 2015]).

11. Outre le fait que le communiqué de l'État islamique confirme cette idée, l'ancien juge antiterroriste Marc Trévidic affirme lors d'une interview sur TF1 le lendemain des attentats, qu'une salle de spectacles, et plus précisément un concert rock, aurait été une cible prévue pour un attentat (" une cible mécréante par excellence») dès le mois d'août 2015 ("Attaques à Paris : Le Bataclan "un cible [sic] par excellence, la musique complètement haram" pour les isl », TF1, 14 novembre 2015, http://lci.tf1.fr/france/attaques-a-paris-le-bataclan-un-cible-par-excellence-lamusique-8683502.html [consulté le 25 novembre 2015]). D'autres analyses mettent en avant le fait que le Bataclan était déjà une cible de certaines organisations djihadistes à cause de la tenue 
répétée de concerts de soutien et de collecte de fonds en faveur de l'armée israélienne (MORTAIGNE, Véronique et GUIBERT, Nathalie, «Le Bataclan, un haut lieu de la culture ciblé de longue date par les islamistes ", Le Monde, 16 novembre 2015 (http://www.lemonde.fr/attaquesa-paris/article/2015/11/15/le-bataclan-un-haut-lieu-de-la-culture-cible-de-longue-date-par-lesislamistes_4810424_4809495.html [consulté le 25 novembre 2015]).

12. «Eagles of Death Metal s'exprime sur les attentats de Paris ", Vice news (https:// news.vice.com/fr/video/eagles-of-death-metal-s-exprime-sur-les-attentats-de-paris [consulté le 27 novembre 2015]).

13. Les portraits des victimes de l'attaque du Bataclan publiés par Le Monde montrent des personnes mélomanes, passionnées de rock, dont une partie pratiquait la musique en amateur ou de façon professionnelle («\#EnMémoire », Le Monde, http://www.lemonde.fr/attaques-a-paris/ visuel/2015/11/25/enmemoire_4817200_4809495.html [consulté le 12 décembre 2015]).

14. Le salafisme - du mot salaf ("ancêtre ») - est un courant rigoriste et puritain de l'islam sunnite qui imposerait aux musulmans le retour aux premières lois de l'islam. Pour une analyse de ce courant et de ses différentes factions, voir WIKTOROWICZ, Quintin, « Anatomy of the Salafi Movement ", Studies in Conflict and Terrorism, vol. 29, $\mathrm{n}^{\circ}$ 3, 2006, p. 207-239. Sur l'implantation du salafisme en France, voir notamment ADRAOUI, Mohamed-Ali, Du Golfe aux banlieues, le salafisme mondialisé, Paris, Puf, 2013.

15. Je considère la propagande comme un dispositif qui implique des stratégies de domination, des discours, des pratiques de socialisation, des objets symboliques et des institutions, et qui viserait non seulement à influencer mais aussi à provoquer l'identification et l'adhésion consciente des individus à un pouvoir perçu comme légitime. Son efficacité résiderait donc dans sa capacité à imposer comme légitimes des croyances fondées sur une idéologie spécifique afin de provoquer une action dans un contexte déterminé. Voir notamment VELASCO-PUFLEAU, Luis, « Reflections on Music and Propaganda", Contemporary Aesthetics, vol. 12, 2014 (http:// quod.lib.umich.edu/c/ca/7523862.0012.007?view=text;rgn=main).

16. «Que nous veut Daesh?», Culturesmonde, émission citée. Il fait aussi le constat que «la propagande du Califat est bien plus efficace que la nôtre ».

17. Pour Nabil Mouline, le djihadisme «est avant tout une idéologie globale. À la faveur du bricolage intellectuel qui résulte du détournement de concepts, de symboles et d'images d'origine musulmane ou européenne, ses dépositaires prétendent offrir aux "croyants" un nouveau départ, une nouvelle identité et un nouveau mode de vie pour réussir ici-bas et dans l'au-delà. En somme, une représentation du monde qui donne la certitude d'appartenir à quelque chose de plus grand que soi : le groupe d'élus chargé par Dieu de rétablir la vraie religion et de réunifier l'oumma (la communauté des croyants) sous l'égide du califat - la monarchie universelle islamique -, avant de se lancer à la conquête du monde et d'obtenir la salut » (MOULINE, Nabil, "Genèse du djihadisme ", Le Monde diplomatique, $\mathrm{n}^{\circ}$ 741, décembre 2015, p. 1). Pour une analyse pluridisciplinaire de ce phénomène, voir BÉNICHOU, David, KHOSROKHAVAR, Farhad, MIGAUX, Philippe, Le Jihadisme. Le comprendre pour mieux le combattre, Paris, Plon, 2015.

18. Ainsi que le montrent les travaux de Dounia Bouzar sur l'embrigadement des jeunes français (voir notamment BOUZAR, Dounia, CAUPENNE, Christophe, VALSAN, Sulayman, «La métamorphose opérée chez le jeune par les nouveaux discours terroristes ", CPDSI, http:// www.bouzar-expertises.fr/images/docs/METAMORPHOSE.pdf [consulté le 23 novembre 2015]).

19. KHOSROKHAVAR, Farhad, Radicalisation, Paris, Éditions de la Maison des Sciences de l'Homme, 2014, p. 74-75.

20. Pour un panorama sur cette question, voir KORPE, Marie, REITOV, Ole et CLOONAN, Martin, "Music Censorship From Plato to the Present», in BROWN, Steven et VOLGSTEN, Ilrik (eds.), Music and Manipulation. On the Social Uses and Social Control of Music, New York/Oxford, Berghahn Books, 2006, p. 239-263. Pour des analyses complémentaires sur la censure ou l'interdiction de la musique dans des contextes divers (notamment en Afghanistan, Corée du Nord, Afrique du Sud, 
Iran, Mali), voir KORPE, Marie (éd.), Shoot the Singer! Music Censorship Today, Londres, Zed Books, 2004 ainsi que MORGAN, Andy, Music, Culture and Conflict in Mali, Copenhague, Freemusic, 2013.

21. Le terme sama « désigne d'abord et avant tout l'écoute passive de la musique et n'indique qu'implicitement l'expression active de la musique et la pratique musicale » (SHILOAH, Amnon, "Judaïsme et islam: les monothéismes face à la musique ", in NATTIEZ, Jean-Jacques (ed.), Musiques. Une encyclopédie pour le XXI ${ }^{e}$ siècle, vol. 3 « Musiques et cultures ", Arles, Actes Sud/Cité de la Musique, 2005, p. 371). Il renvoie à la musique sacrée et religieuse, s'opposant au terme ghina, « qui connote la musique profane et savante, mais englobe également la musique rurale ou d'autres formes simples, comme le vieux chant bédouin du dromadaire » (SHILOAH, Amnon, La Musique dans le monde de l'islam, Paris, Fayard, 2002, p. 77).

22. Id.

23. Id.

24. Ibid., p. 77-78. Toujours selon cet auteur, l'interdiction absolue de la musique ne toucherait que « l'art savant, qui fait étalage de la vanité humaine et de ses intérêts primesautiers pour les mondanités. Toutefois, cet interdit ne s'applique pas aux mélodies populaires ou rurales ni par extension, à certaines formes en usage dans la musique religieuse qui ne sont pas considérées comme musique per se et ne sont pas désignées par le terme de musique. Cet état de choses peut s'expliquer par la prédominance de la parole sur la musique dans le domaine tant religieux que folklorique. C'est ici que la combinaison des sons est reléguée à un rôle secondaire ; elle se révèle en effet un schéma principalement confectionné pour mettre en évidence les mots et faciliter la compréhension du texte» (ibid., p. 89).

25. Le Coran n'évoquant pas la musique (ghina) de manière explicite, les opposants à celle-ci vont notamment surinterpréter le verset 6 de la sourate (chapitre) Luqman (XXXI) afin de justifier leur point de vue. Ainsi, ils vont associer la musique aux «discours plaisants » [ou "paroles futiles»] dont fait mention le Coran : «Et, parmi les hommes, il en est qui, dénués de science, achètent de plaisants discours pour égarer hors du chemin d'Allah et pour le prendre en raillerie. Ceux-là subiront un châtiment avilissant ». Voir, SHILOAH, Amnon, La Musique dans le monde de l'islam, op. cit., p. 78 ; Al-ALBANI, Nasir Al-Din, La musique en Islam. Chants soufis, Anashid islamiques et les instruments de musique, Bruxelles, Al-Hadith, 2013, p. 91-93.

26. Pour des exemples précis concernant ces sociétés sécrètes voir BOUZAR, Dounia, Comment sortir de l'emprise « djihâdiste » ?, Ivry-sur-Seine, Éditons de l'Atelier, 2015, p. 42-48.

27. Cette conception complotiste de la société est enracinée dans la vision djihadiste du monde qui opposerait les mécréants aux musulmans ; vision prônée, par exemple, par Al-Qaïda, comme l'écrit Anwar Al-Awlaki : "Chers frères et sœurs la question est urgente, aujourd'hui notre ennemi est sans nation, ni race. C'est un système kufr [mécréant] avec une portée mondiale. Les kuffar [mécréants] conspirent aujourd'hui contre nous comme jamais auparavant. Donc pourrions-nous nous diriger vers la grande bataille entre les Romains et les Musulmans - Al Malhamah - dont le Prophète (saaws) a parlé ?», AL-AWLAKI, Anwar, 44 Ways to Support Jihad, Victorious Media, [s.d.], p. 2.

28. « La musique est Haram (illicite) en Islam », https://www.youtube.com/watch?v=FGoriapzFrE (mis en ligne le 3 septembre 2012, consulté le 18 novembre 2015).

29. KHOSROKHAVAR, Farhad, Radicalisation, op. cit., p. 154-155.

30. L'analyse de l'intertextualité des images avec les chants et le discours parlé mériterait une étude pluridisciplinaire approfondie, que le format de cet article ne permet pas.

31. "Pourquoi Allah a t'il interdit la music [sic]», https://www.youtube.com/watch?v=uyFWfRuMVA (mis en ligne le 16 novembre 2014, consulté le 18 novembre 2015). Sauf indication contraire, toutes les citations qui suivent sont extraites de cette vidéo.

32. Le théologien salafiste Nasir Al-Din Al-Albani va encore plus loin en affirmant que «la musique procure un enivrement plus grand que celui du vin, ses adeptes y trouvent une jouissance sans pareil [...]. Cela les détourne du rappel d'Allah et de la prière davantage que le 
font les boissons enivrantes, cela engendre plus d'inimité et de haine entre eux que les boissons enivrantes, au point que l'un tue l'autre sans même le toucher de la main mais avec les démons qui l'accompagnent. Ils connaissent des états démoniaques car les démons viennent à eux dans cette situation » (Al-ALBANI, Nasir Al-Din, La musique en Islam. Chants soufis, Anashid islamiques et les instruments de musique, op. cit., p. 113).

33. Les salafistes qualifient les personnes qui n'adhèrent pas à leur vision dogmatique et « puriste » de l'islam d'hypocrites, d'égarés, d'hérétiques ou de mécréants.

34. Les thèmes de l'incompatibilité de la musique avec l'islam et du complot satanique de l'industrie du disque sont repris et déclinés dans de nombreuses vidéos, par exemple : "Écoutez ce frère svp / Islam musique haram ", https://www.youtube.com/watch?v=VXiock2IPGQ (mise en ligne le 14 mars 2012, consulté le 24 novembre 2015) ainsi que «La musique est strictement interdit [sic] en Islam!», https://www.youtube.com/watch?v=cFMzAk5zGzo (mis en ligne le 6 juillet 2012, consulté le 24 novembre 2015).

35. «Pourquoi Allah a t'il interdit la music [sic]», https://www.youtube.com/watch?v=uyFWfRuMVA (consulté le 18 novembre 2015).

36. Ceci renforce la frontière symbolique entre croyants et mécréants, le principe « al-wala wa' albara » (" l'allégeance et la rupture ») : « le croyant doit une fidélité et une loyauté absolues à tous les autres membres de la communauté. En revanche, les relations avec les mécréants se limitent théoriquement à la conversion, la soumission ou la guerre » (MOULINE, Nabil, "Genèse du djihadisme ", Le Monde diplomatique, op. cit., p. 15).

37. «Écouter la musique encourt le risque d'être transformé en singe ou porc!!! », https:// www.youtube.com/watch?v=5wpBb7Ulqoc (mis en ligne le 21 septembre 2015, consulté le 18 novembre 2015). Cette courte vidéo constitue un montage des extraits d'un de ses cours coraniques pour enfants. Dans une autre vidéo, il réaffirme l'idée selon laquelle la musique serait « le son de Shaytan [le diable], la voix de Shaytan, l'incantation de la fornication », voir « Islam La Musique - La voix de Shaytan (du diable) par Rachid Abou Houdeyfa ", https:// www.youtube.com/watch?v=VaMEdzdaw8M (mis en ligne le 22 janvier 2012, consulté le 24 novembre 2015).

38. «Écouter la musique encourt le risque d'être transformé en singe ou porc!!! », https:// www.youtube.com/watch? $\mathrm{v}=5$ wpBb7Ulqoc (consulté le 18 novembre 2015).

39. SEMELIN, Jacques, Purifier et détruire. Usages politiques des massacres et génocides, Paris, Éd. du Seuil, 2005, p. 58.

40. D'après la philosophe Chantal Mouffe, la relation "Nous/Eux", nécessaire dans toute construction identitaire, se transforme dans une relation "ami-ennemi », lorsque «les autres, considérés jusque-là comme étant simplement différents, sont perçus comme s'ils remettaient en cause notre identité et menaçaient notre existence" (MOUFFE, Chantal, Agonistique. Penser politiquement le monde, Paris, Beaux-Arts de Paris éditions, 2014, p. 27).

41. SEMELIN, Jacques, Purifier et détruire. Usages politiques des massacres et génocides, op. cit., p. 58.

42. Voir KRISTANADJAJA, Gurvan, "L'imam de Brest, un "salafiste YouTube" au rôle ambigu ", Libération, 23 novembre 2015 (http://www.liberation.fr/france/2015/11/23/l-imam-de-brest-unsalafiste-youtube-au-role-ambigu_1415599 [consulté le 10 décembre 2015]).

43. "L'imam R. Abou Houdeyfa sur la musique, les femmes...", https://www.youtube.com/ watch?v=cZG-7G1DvVA (mis en ligne le 24 septembre 2015, consulté le 24 novembre 2015).

44. Id.

45. Id.

46. Le wahhabisme est un mouvement politico-religieux saoudien, fondé au XVIII ${ }^{\mathrm{e}}$ siècle par Mohammed ben Abdelwahhab. Il s'agit d'un courant fondamentaliste issue de l'islam sunnite hanbalite qui partage le corpus idéologique du salafisme. Sur le wahhabisme, voir notamment MOULINE, Nabil, Les Clercs de l'islam. Autorité religieuse et pouvoir politique en Arabie Saoudite (XVIII XXI siècle), Paris, Puf, 2011. 
47. Selon Hasna Hussein, « la période postprintemps arabe se caractérise aussi par l'émergence de nouvelles chaînes satellitaires islamiques tenues par les salafistes à partir de 2011 ». Ces chaînes transnationales prétendent dépasser les clivages nationaux par le traitement de l'actualité régionale (notamment du Maghreb et du Moyen-Orient) sous un angle généraliste ou spécialisé (HUSSEIN, Hasna, "La télévision et la transformation de l'éthique islamiste: les présentatrices niqabées sur les chaînes salafistes satellitaires arabes ", Recherches féministes, vol. 28, $\mathrm{n}^{\circ} 2,2015$, p. 223-242).

48. «La musique est haram! », https://www.youtube.com/watch?v=tqqeQpGL7lo (mis en ligne le 3 septembre 2012, consulté le 18 novembre 2015).

49. http://alrahma.tv

50. « La musique est Haram (illicite) en Islam », https://www.youtube.com/watch?v=FGoriapzFrE (consulté le 18 novembre 2015).

51. Cette distinction est loin d'être spécifique à l'islam. Au Mexique, par exemple, le peuple Wixarika nomme le chant rituel du mara'kame (chaman) wawi niuki, «la parole qui lie [aux ancêtres] », et ne le considère pas comme une pratique relevant de la "musique » (LEMESTRE, Denis, Le Chamane et son chant. Relations ethnographiques d'une expérience parmi les Huicholes du Mexique, Paris, L'Harmattan, 2003, p. 196).

52. «Anasheed Halal ou Haram, Mohammed Hassan», https://www.youtube.com/watch? $\mathrm{v}=\mathrm{vAofyBu07AI}$ (mis en ligne le 22 février 2014, consulté le 18 novembre 2015).

53. «Ouléma » est un titre donné par les musulmans aux docteurs de la loi coranique (juristes et théologiens), garants du respect et de l'application des principes de l'islam. Le Conseil des grands oulémas est une institution religieuse composée d'une vingtaine de théologiens, formellement nommés par le roi.

54. Avis juridique donné par un spécialiste de la loi islamique sur une question particulière.

55. SAID, Behnam, "Hymns (Nasheeds): A Contribution to the Study of the Jihadist Culture", Studies in conflict and Terrorism, vol. 35, $\mathrm{n}^{\circ} 12,2012, \mathrm{p} .869$.

56. SHILOAH, Amnon, La Musique dans le monde de l'islam, op. cit., p. 29-30.

57. Said propose quatre catégories principales d'anasheed djihadistes : chants de bataille (battle hymns), chants de martyrs (martyr hymns), chants de deuil (mourning hymns) et chants de louange (praising hymns). Soulignant le lien direct entre les anasheed djihadistes et la kasidah (poésie) néoclassique et anticolonialiste, il fait remonter l'essor de ce genre aux années 1980, dans le contexte des luttes des groupes armés de mouvance djihadiste en Afghanistan et en Palestine, puis en Irak et en Tchétchénie.

58. AL-ALBANI, Nasir Al-Din, Tahrim Alat at-Tarab, Maktaba Al-Dalil, 1996, p. 181 [cité dans SAID, Behnam, « Hymns (Nasheeds): A Contribution to the Study of the Jihadist Culture », op. cit., p. 869].

59. SAID, Behnam, « Hymns (Nasheeds): A Contribution to the Study of the Jihadist Culture », loc. cit.

60. Id.

61. Al-ALBANI, Nasir Al-Din, La musique en Islam. Chants soufis, Anashid islamiques et les instruments de musique, op. cit., p. 80.

62. AL-AWLAKI, Anwar, 44 Ways to Support Jihad, op. cit., p. 2.

63. «40. Nasheeds: Muslims need to be inspired to practice Jihad. In the time of Rasulullah (saaws) he had poets who would use their poetry to inspire the Muslims and demoralize the disbelievers. Today nasheed can play that role. A good nasheed can spread so widely it can reach to an audience that you could not reach through a lecture or a book. Nasheeds are especially inspiring to the youth, who are the foundation of Jihad in every age and time. Nasheeds are an important element in creating a "Jihad culture." Nasheeds are abundant in Arabic bur scarce in English. Hence it is important for talented poets and talented singers to take up this responsibility. The nasheeds can cover topics such as: Martyrdom, Jihad is our only solution, support of the present day leaders of Jihad (to connect the youth to them), the situation of the 
Ummah, the responsibility of the youth, the victory of Islam and defending the religion. The nasheeds should focus on Justice rather than peace and strength rather than weakness. The nasheeds should be strong and uplifting and no apologetic and feminine » (Ibid., p. 19).

64. «A device for clarifying social order, for structuring subjectivity (desire and the temporal parameters of emotion and the emotive dimension of interaction) and for establishing a basis for collaborative action" (la traduction est de l'auteur; DE NORA, Tia, Music in everyday life, Cambridge, Cambridge University Press, 2000, p. 111 [cité dans PIESLAK, Jonathan, Sound Targets. American Soldiers and Music in the Iraq War, Bloomington, Indiana University Press, 2009, p. 54]).

65. Pour une analyse de la place des anasheed dans la culture musicale et la propagande d'AlQaïda, voir PIESLAK, Jonathan, Radicalism and Music, Middletown, Wesleyan University Press, 2015, p. 14-44.

66. Pour un aperçu de la vaste production d'anasheed djihadistes de l'État islamique, voir la rubrique Ajnad Media du site Jihadology : http://jihadology.net/category/ajnad-foundation-formedia-production (consulté le 28 novembre 2015).

67. Dont les revues Dabiq, Islamic State News et Islamic State Report (en anglais) ou Dar al-Islam (en français). Pour un exemple de vidéos de propagande produites par le Al-Hayat Media Center, voir "And no Respite», http://jihadology.net/2015/11/24/new-video-message-from-the-islamicstate-and-no-respite (consulté le 30 novembre 2015).

68. Par sa thématique générale et certains des sujets qu'il aborde, le nasheed djihadiste peut être mis en parallèle avec la chanson de croisade du XIII ${ }^{\mathrm{e}}$ siècle, genre « créé pour inviter les chrétiens à participer aux guerres de reconquête des Lieux saints. Accumulant les arguments en ce sens, elles pouvaient même quelquefois évoquer la colère divine au jour du Jugement dernier contre ceux qui auraient commis la lâcheté de refuser le départ. [...] Dans ce petit corpus, l'intérêt porté au texte prime le plus souvent sur la valeur mélodique " (DE MONTALEMBERT, Eugène et ABROMONT, Claude, Guide des genres de la musique occidentale, Paris, Fayard/Henry Lemoine, 2010, p. 217-218). Pour un exemple éclairant de ce genre, voir «Musique au temps de Saint Louis. Chanson de croisade ", https://www.youtube.com/watch?v=oTUvdemWYzE (mis en ligne le 2 mai 2014, consulté le 10 décembre 2015).

69. "Communiqué sur l'attaque bénie de Paris contre la France croisée", https:// ia801505.us.archive.org/32/items/CommuniqueAudio/communique\%20audio.mp3 (mis en ligne le 14 novembre 2015, consulté le 15 novembre 2015).

70. SEELOW, Soren et GUIBERT, Nathalie, "Fabien Clain, la "voix" du massacre de Paris, avait déjà menacé le Bataclan en 2009 ", Le Monde, 18 novembre 2015 (http://www.lemonde.fr/ attaques-a-paris/article/2015/11/18/fabien-clain-la-voix-du-massacre-de-paris-avait-deja-

menace-le-bataclan-en-2009_4812298_4809495.html?xtmc=voix_communique_attentats\&xtcr=14

[consulté le 28 novembre 2015]) ; BUI, Doan, « De Toulouse à la Syrie : Fabien Clain, le parrain djihadiste, qui a revendiqué les attentats", L'Obs, 19 novembre 2015 (http:// tempsreel.nouvelobs.com/attentats-terroristes-a-paris/20151118.0BS9734/de-toulouse-a-lasyrie-fabien-clain-le-parrain-djihadiste.html [consulté le 28 novembre 2015]).

71. BUI, Doan, « De Toulouse à la Syrie : Fabien Clain, le parrain djihadiste, qui a revendiqué les attentats ", op. cit.

72. Il est fort possible que les combattants de l'État islamique aient chanté ce nasheed avant les attaques de Paris. Comme l'a montré Jonathan Pieslak dans ses recherches sur la musique dans la guerre d'Irak, chanter ensemble constitue un moment privilégié des rituels des soldats étatsuniens et des combattants irakiens aussi bien avant que durant le combat, voir PIESLAK, Jonathan, Sound Targets. American Soldiers and Music in the Iraq War, op. cit., p. 74-75.

73. Il est possible que le nasheed «Avance, avance » ait été enregistré en studio par une seule personne utilisant la technique du re-recording, c'est-à-dire l'enregistrement successif d'une piste audio sur une autre. Quoi qu'il en soit, l'enregistrement de ce nasheed requiert des compétences musicales et des connaissances techniques certaines. 
74. En accord avec l'idéologie de l'État islamique : «Celui qui a l'expérience du djihad sait que ce n'est rien d'autre que violence, cruauté, terrorisme, frayeur et massacre » (NAJI, Abu Bakr, The Management of Savagery. The Most Critical Stage Through Which the Umma Will Pass, op. cit., p. 31).

75. La même forme est utilisée par l'État islamique dans le communiqué audio de revendication de l'attaque de San Bernardino (Californie, le 2 décembre 2015), diffusé sur Internet le 5 décembre 2015.

76. La taille d'un ficher audio stéréo de trois minutes en qualité d'enregistrement standard $(44,1 \mathrm{KHz}, 16$ bits) est d'environ 30 mégaoctets (Mo). La compression du fichier en mp3 à $128 \mathrm{kbps}$ permet de réduire la taille à moins de $3 \mathrm{Mo}$. Comme l'a formulé Jonathan Sterne, « Le but du mp3 est de rendre les fichiers audio plus petits grâce à la compression de données, de sorte qu'ils soient plus faciles à échanger dans un contexte où la bande passante est limitée, comme sur Internet, et plus faciles à stocker dans un contexte où l'espace des données est limité, comme sur un disque dur » [The point of mp3s is to make audio files smaller through data compression so that they are easier to exchange in a limited bandwidth environment such as the internet, and easier to store in a limited dataspace environment, such as a hard drive], STERNE, Jonathan, "The mp3 as cultural artifact ", New Media and Society, vol. 8, n 5, 2006, p. 828.

77. La vidéo est diffusée sur internet le 3 février 2015. La cruauté de cette exécution reflète la stratégie de terreur suivie par l'État islamique: «La politique de la violence doit également être poursuivie de telle sorte que si les demandes ne sont pas satisfaites, l'otage doit être liquidé d'une manière terrifiante, qui sèmera l'effroi dans les cœurs de l'ennemi et de ceux qui le soutiennent » (NAJI, Abu Bakr, The Management of Savagery. The Most Critical Stage Through Which the Umma Will Pass, op. cit., p. 33).

78. Pour une traduction en anglais de la version arabe de ce nasheed, voir http:// www.aymennjawad.org/2015/02/new-nasheed-from-islamic-state-ajnad-media-soon (consulté le 28 novembre 2015). L'État islamique a également diffusé sur Internet un vidéoclip de ce nasheed (sous-titré en anglais et intitulé "Soon, soon»), consultable ici : https://archive.org/details/ SoonSoon (mis en ligne le 9 décembre 2015, consulté le 12 décembre 2015).

79. La réalisation de cette vidéo de 22 minutes montre que l'État islamique possède de ressources techniques considérables ainsi que d'une équipe professionnelle de production. Le tournage utilise au moins deux caméras et présente un travail soigné sur la lumière et le son. Le travail de postproduction et d'effets spéciaux est aussi très important (modélisation, animation et particules en 3D, morphing, incrustations, bruitage), requérant sans doute le travail de toute une équipe pendant plusieurs semaines.

80. «Healing the believers' chests», https://archive.org/details/ AlFurqaanMediaHealingTheBelieversChests (mis en ligne le 15 février 2015, consulté le 8 décembre 2015).

81. L'État islamique entend rendre justice aux familles des victimes en [lui] faisant subir les mêmes souffrances que celles endurées par les civils morts sous ses bombes. Voir « Burning of the Murtadd Pilot », Dabiq, ${ }^{\circ}$ 7, février 2015, p. 5-8.

82. Comme dans la série de trois vidéos « Killing the Apostates in Revenge For the Monotheists Wilayat Khurasan»: première partie http://jihadology.net/2015/06/18/new-video-messagefrom-the-islamic-state-killing-the-apostates-in-revenge-for-the-monotheists-1-wilayat-khurasan (mis en ligne le 18 juin 2015, consulté le 8 décembre 2015) ; seconde partie http://jihadology.net/ 2015/08/09/new-video-message-from-the-islamic-state-killing-the-apostates-in-revenge-for-

the-monotheists-2-wilayat-khurasan (mis en ligne le 9 août 2015, consulté le 8 décembre 2015); troisième partie http://jihadology.net/2015/12/07/new-video-message-from-the-islamic-statekilling-the-apostates-in-revenge-for-the-monotheists-3-wilayat-khurasan (mis en ligne le 7 décembre 2015, consulté le 8 décembre 2015).

83. «Que nous veut Daesh?», Culturesmonde, émission citée. 
84. DE NORA, Tia, "Quand la musique de fond entre en action », Terrain, $n^{\circ} 37,2001$ (http:// terrain.revues.org/1310).

85. La musique peut à l'inverse avoir un rôle important dans le désembrigadement des jeunes, en tant que déclencheur émotionnel pour les ramener à leurs anciens repères en ravivant de bons souvenirs (voir BOUZAR, Dounia, Comment sortir de l'emprise « djihâdiste» ?, op. cit., p. 109-120).

86. MOUFFE, Chantal, Agonistique. Penser politiquement le monde, op. cit., p. 113.

87. SALAZAR, Philippe-Joseph, Paroles armées. Comprendre et combattre la propagande terroriste, Paris, Lemieux Éditeur, 2015, p.131. Cet ouvrage développe une analyse approfondie de la propagande de l'État islamique.

88. Par exemple, lors du Congrès du Parlement français qui s'est tenu au Château de Versailles le 16 novembre 2015 : "Congrès à Versailles: "La Marseillaise" chantée par les parlementaires après les discours de Hollande ", Francetvinfo, 16 novembre 2015, http://www.francetvinfo.fr/ faits-divers/terrorisme/attaques-du-13-novembre-a-paris/video-la-marseillaise-entonnee-aucongres-a-versailles_1178631.html (consulté le 10 décembre 2015).

89. Par exemple, lors du match amical entre l'Angleterre et la France du 17 novembre 2015 : "Angleterre-France : Wembley chante en chœur la "Marseillaise" en hommage aux victimes des attentats », Francetvinfo, 17 novembre 2015, http://www.francetvinfo.fr/faits-divers/ terrorisme/attaques-du-13-novembre-a-paris/video-anglettere-france-wembley-chante-enchoeur-la-marseillaise-en-hommage-aux-victimes-des-attentats_1180347.html (consulté le 10 décembre 2015).

90. Par exemple, au Metropolitan Opera de New-York, le 15 novembre 2015 (« The French hymn resounds to the Metropolitan Opera de NYC», https://www.youtube.com/watch? v=l6vGq_rHAmE [mis en ligne le 15 novembre 2015, consulté le 10 décembre 2015]), à la cathédrale Notre-Dame de Paris, le 15 novembre 2015 ( « La Marseillaise retentit à Notre-Damede-Paris (15 novembre 2015) », https://www.youtube.com/watch?v=Pa3YOKk-uLk [mis en ligne le 15 novembre 2015, consulté le 10 décembre 2015]) ou lors d'un concert de Madonna à Bercy (Paris), le 9 décembre 2015 («Madonna - La Marseillaise - Live @ Paris (Bercy), 9/12/2015», https://www.youtube.com/watch?v=AhwyG9XTjls [mis en ligne le 9 décembre 2015, consulté le 10 décembre 2015]).

91. "Paris s'est effondré ", voir http://jihadology.net/2015/11/20/new-video-message-fromthe-islamic-state-paris-has-collapsed-wilayat-\%E1\%B8\%A5alab (mis en ligne le 20 novembre 2015, consulté le 29 novembre 2015). S'appuyant sur la rhétorique sonore du nasheed, un combattant français de l'État islamique qualifie dans cette vidéo les attentats de Paris de «très bonne nouvelle pour nous » et déclare : « Nous répondons juste à votre agression, c'est vous les terroristes, vous mentez dans les médias sur nous. Quand vous venez dans les pays musulmans c'est vous qui violez, c'est vous qui volez, c'est vous qui tuez. Nous, on se défend ».

92. SALAZAR, Philippe-Joseph, Paroles armées. Comprendre et combattre la propagande terroriste, op. cit., p. 93.

\section{RÉSUMÉS}

Lors de la série d'attaques terroristes simultanées qui ont eu lieu à Paris le vendredi 13 novembre 2015, trois combattants de l'État islamique prennent d'assaut la salle de concert du Bataclan où se tenait un concert de rock. Cet article propose une analyse du rapport entre la portée symbolique de cette attaque, le discours salafiste sur la musique et l'importance que l'État islamique donne 
au chant dans sa propagande djihadiste. Il montre de quelle façon l'utilisation du nasheed «Avance, avance » dans le communiqué audio de revendication des attentats, vise à ritualiser sa propagande, légitimer la violence, encourager ses combattants et démoraliser son ennemi. Par l'examen des enjeux des pratiques musicales au sein du dispositif de propagande de l'État islamique, ce texte se donne pour objectif de contribuer à une meilleure compréhension de la mécanique idéologique mobilisée par cette organisation djihadiste.

During the series of simultaneous terrorist attacks that took place in Paris on Friday 13 November 2015, three Islamic State fighters stormed the Bataclan concert hall which was holding a rock concert. This article analyses the relationship between the symbolic significance of this attack, the Salafist discourse on music, and the importance of chanting in the Islamic State jihadist propaganda. It shows how the use of nasheed "Avance, avance" in the audio statement claiming responsibility for the attacks, ritualises propaganda, legitimises violence, motivates its fighters, and demoralises the enemy. By examining the issue of musical practices and their mobilisation within the Islamic State propaganda dispositif, this text aims to further a better understanding of the ideology of this jihadist organization.

\section{INDEX}

Mots-clés : musique, anasheed, terrorisme, violence, État islamique, djihad, émotions, propagande.

Keywords : music, anasheed, terrorism, violence, Islamic State, jihad, emotions, propaganda.

\section{AUTEUR}

\section{LUIS VELASCO-PUFLEAU}

Docteur en Musique et musicologie (Université Paris-Sorbonne), Luis Velasco-Pufleau est chercheur post-doctorant à l'Université de Salzbourg (2013-2016) et Balzan Visiting Fellow à l'Université d'Oxford (2015-2016). Il est également chercheur associé au Centre de recherches sur les arts et le langage (CRAL) de l'École des hautes études en sciences sociales (EHESS). Ses recherches portent notamment sur les rapports entre musique, esthétique et politique au $\mathrm{XX}^{\mathrm{e}}$ et XXI ${ }^{\mathrm{e}}$ siècle. 